\title{
1 \\ Introduction to the Theory of Elastic Waves
}

\subsection{Elastic waves}

Elastic waves are mechanical waves propagating in an elastic medium as an effect of forces associated with volume deformation (compression and extension) and shape deformation (shear) of medium elements. External bodies causing these deformations are called wave sources. Elastic wave propagation involves exciting the movement of medium particles increasingly distant from the wave source. The main factor differentiating elastic waves from any other ordered motion of medium particles is that for small disturbances (linear approximation) elastic wave propagation does not result in matter transport.

Depending on restrictions imposed on the elastic medium, wave propagation may vary in character. Bulk waves propagate in infinite media. Within the class of bulk waves one can distinguish longitudinal waves (compressional waves) and shear waves. A three-dimensional medium bounded by one surface allows for propagation of surface waves (Rayleigh waves and Love waves). Propagation of bulk waves and surface waves is used for describing seismic wave phenomena. Bounding the elastic medium with two equidistant surfaces causes compressional waves and shear waves to interact, which results in the generation of Lamb waves. One can say that a free boundary restricting an elastic body guides and drives waves; therefore the term

Guided Waves in Structures for SHM: The Time-Domain Spectral Element Method, First Edition.

Wieslaw Ostachowicz, Pawel Kudela, Marek Krawczuk and Arkadiusz Zak.

(C) 2012 John Wiley \& Sons, Ltd. Published 2012 by John Wiley \& Sons, Ltd. 
guided waves is also used. Lamb waves and guided waves are used in broadly considered diagnostics and nondestructive testing. There are also waves that propagate on media boundary (interface waves) with names derived from their discoverers: in the interface between two solids Stoneley waves propagate, while in the one between a solid and a liquid Scholte waves propagate.

\subsubsection{Longitudinal Waves (Compressional/Pressure/ Primary/P Waves)}

Longitudinal waves are characterised by particle motion alternately of compression and stretching character. The direction of medium point motion is parallel to the direction of wave propagation (i.e. longitudinal).

\subsubsection{Shear Waves (Transverse/Secondary/S Waves)}

Shear waves are characterised by transverse particle movements in alternating direction. The direction of medium particle motion is perpendicular to

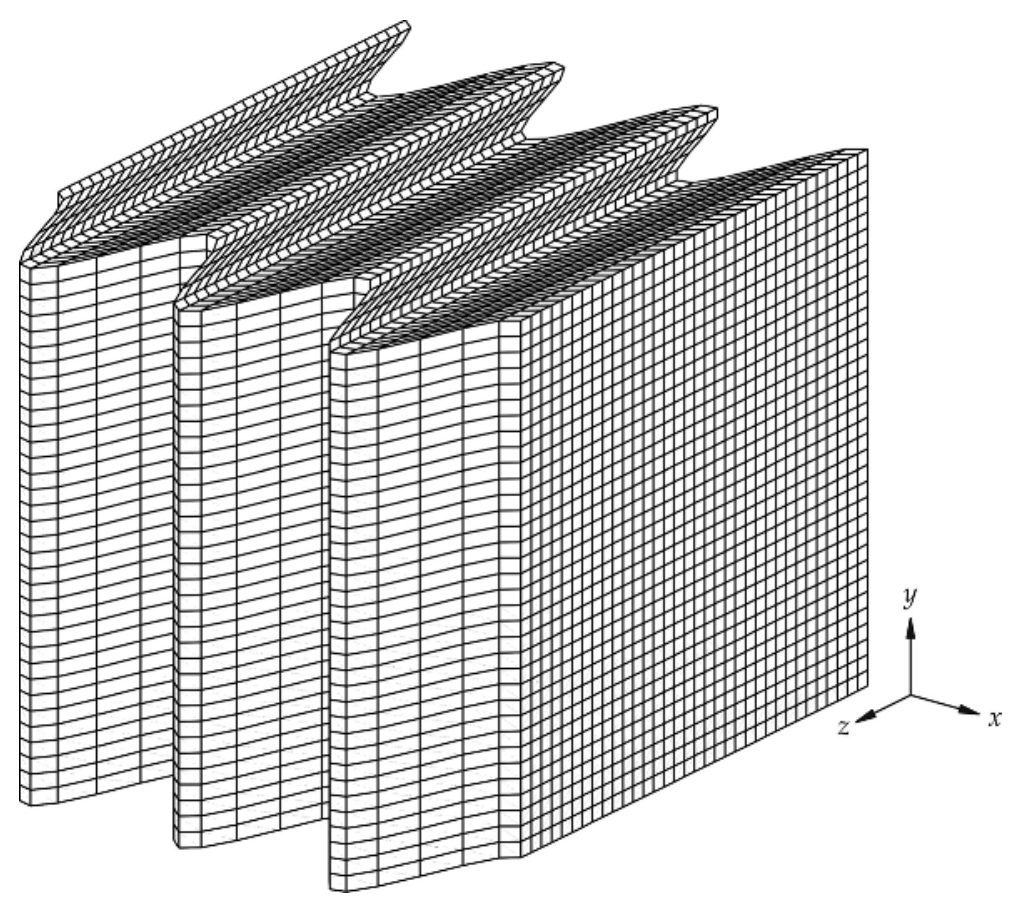

Figure 1.1 Distribution of displacements for the horizontal shear wave 


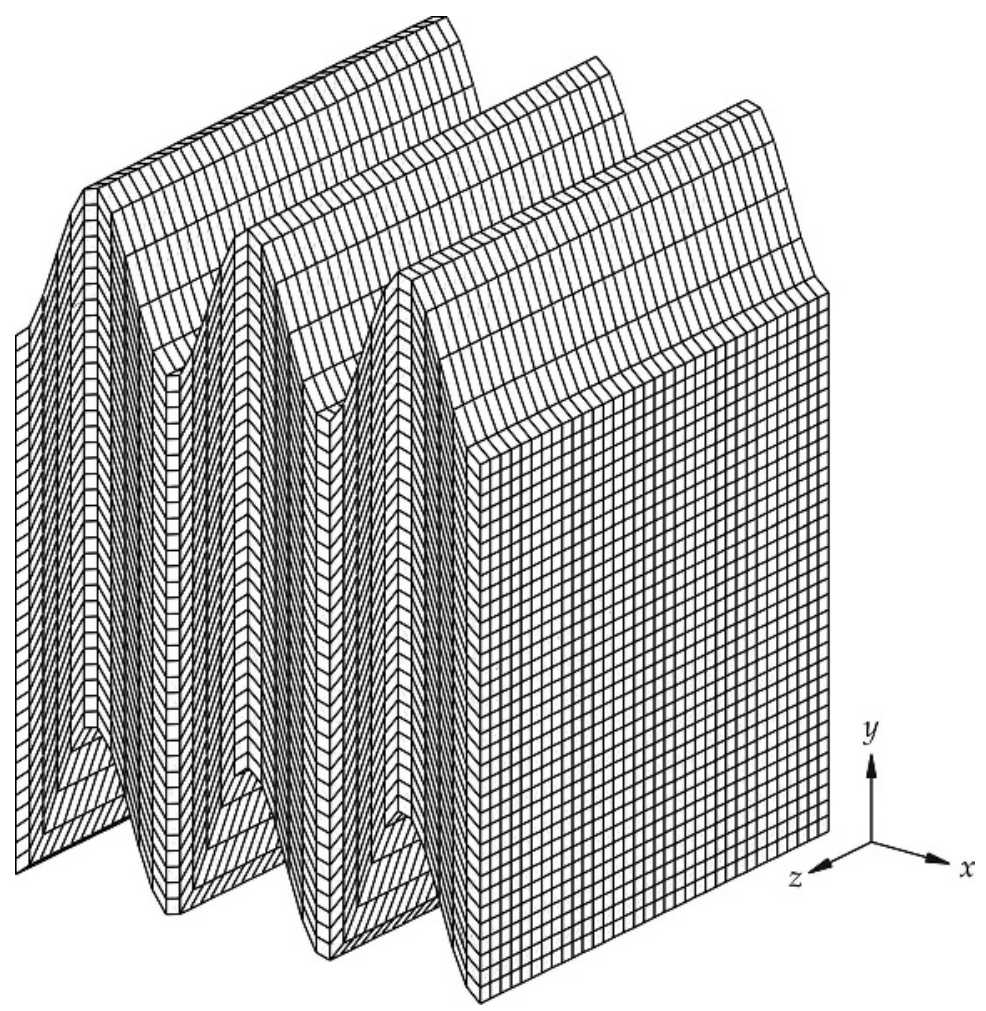

Figure 1.2 Distribution of displacements for the vertical shear wave

the propagation direction (transverse). The transverse particle movement can occur horizontally (horizontal shear wave, $\mathrm{SH}$; see Figure 1.1) or vertically (vertical shear wave, SV; see Figure 1.2).

\subsubsection{Rayleigh Waves}

Rayleigh waves (Figure 1.3) are characterised by particle motion composed of elliptical movements in the $x y$ vertical plane and of motion parallel to the direction of propagation (along the $x$ axis). Wave amplitude decreases with depth $y$, starting from the wave crest. Rayleigh waves propagate along surfaces of elastic bodies of thickness many times exceeding the wave height. Sea waves are a natural example of Rayleigh waves. 


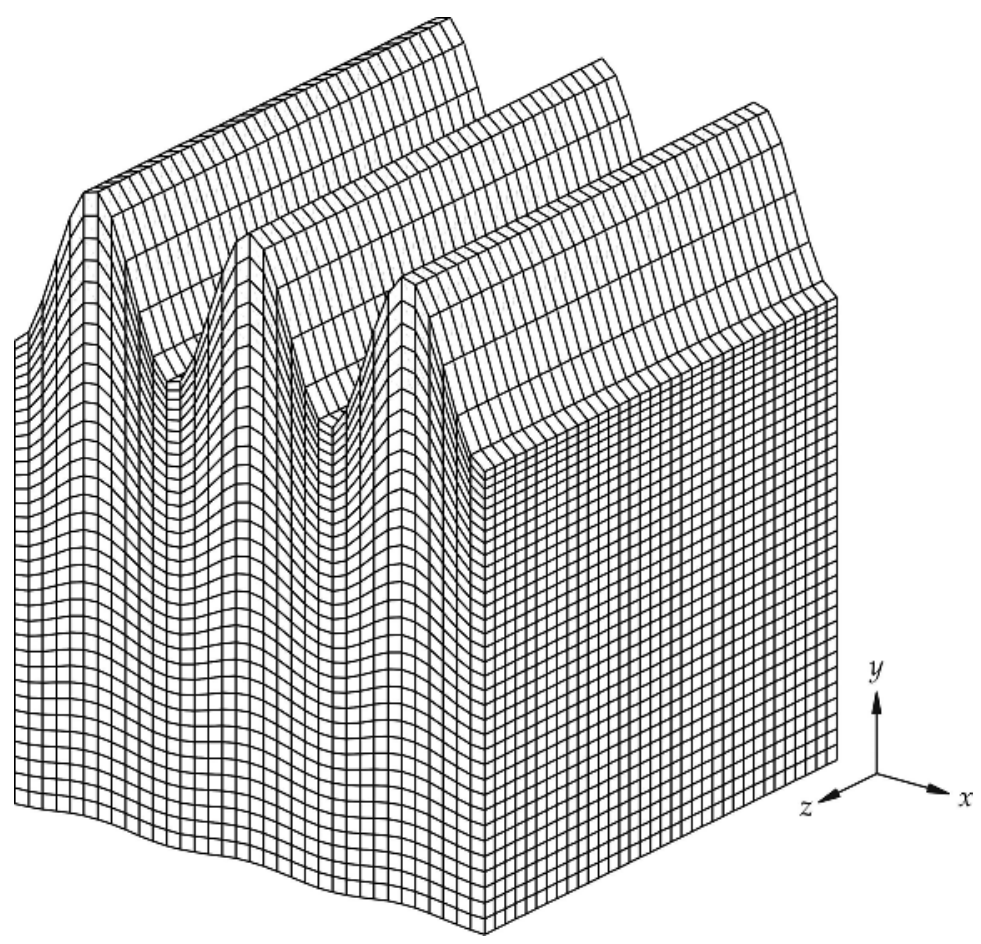

Figure 1.3 Distribution of displacements for the Rayleigh wave

\section{1.4 Love Waves}

Love waves (Figure 1.4) are characterised by particle oscillations involving alternating transverse movements. The direction of medium particle oscillations is horizontal (in the $x z$ plane) and perpendicular to the direction of propagation. As in the case of Rayleigh waves, wave amplitude decreases with depth.

\subsubsection{Lamb Waves}

These waves were named after their discoverer, Horace Lamb, who developed the theory of their propagation in 1917 [1]. Curiously, Lamb was not able to physically generate the waves he discovered. This was achieved by Worlton [2], who also noticed their potential usefulness for damage detection. Lamb waves propagate in infinite media bounded by two surfaces and arise as a result of superposition of multiple reflections of longitudinal $\mathrm{P}$ waves 


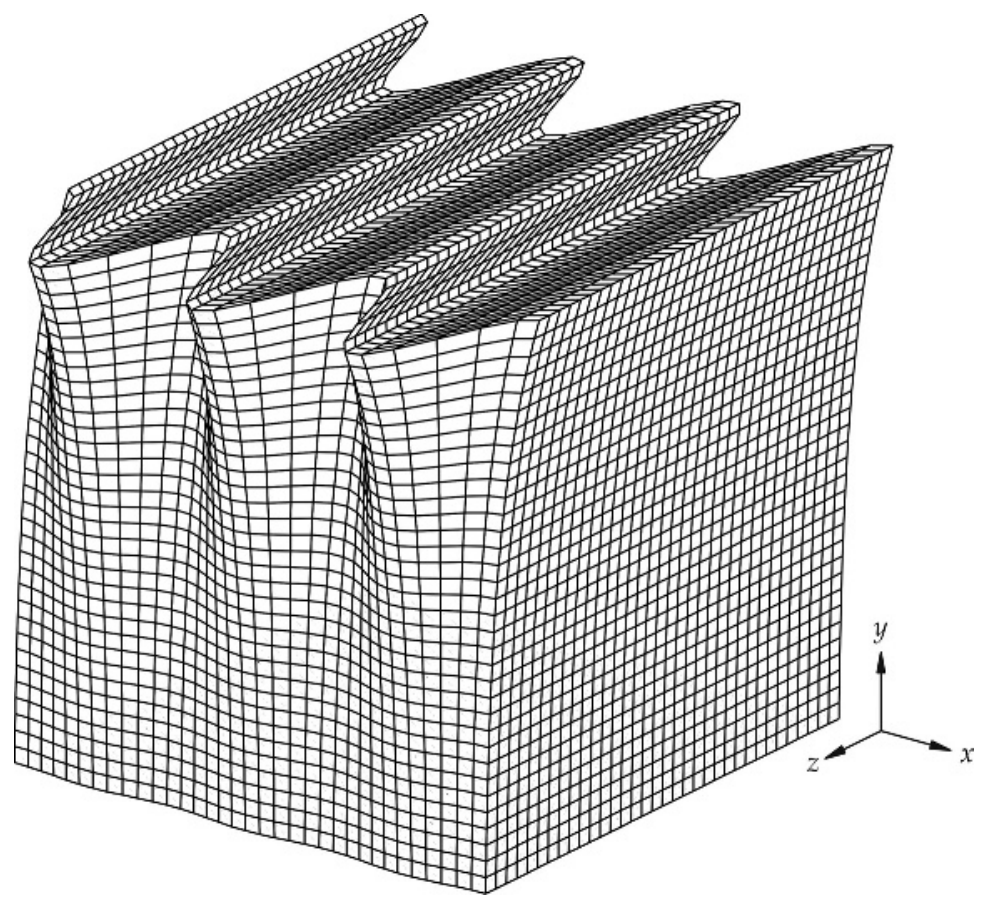

Figure 1.4 Distribution of displacements for the Love wave

and shear SV waves from the bounding surfaces. In the case of these waves medium particle oscillations are very complex in character. Depending on the distribution of displacements on the top and bottom bounding surface, two forms of Lamb waves appear: symmetric, denoted as $S_{0}, S_{1}, S_{2}, \ldots$, and antisymmetric, denoted as $A_{0}, A_{1}, A_{2}, \ldots$ It should be noted that numbers of these forms are infinite. Displacement fields of medium points for the fundamental symmetric mode $S_{0}$ and fundamental antisymmetric mode $A_{0}$ of Lamb waves are illustrated in Figures 1.5 and 1.6, respectively.

\subsection{Basic Definitions}

A specific case of waves as harmonic initial perturbations is considered here:

$$
u(x, 0)=U_{0} \cos (k x)
$$




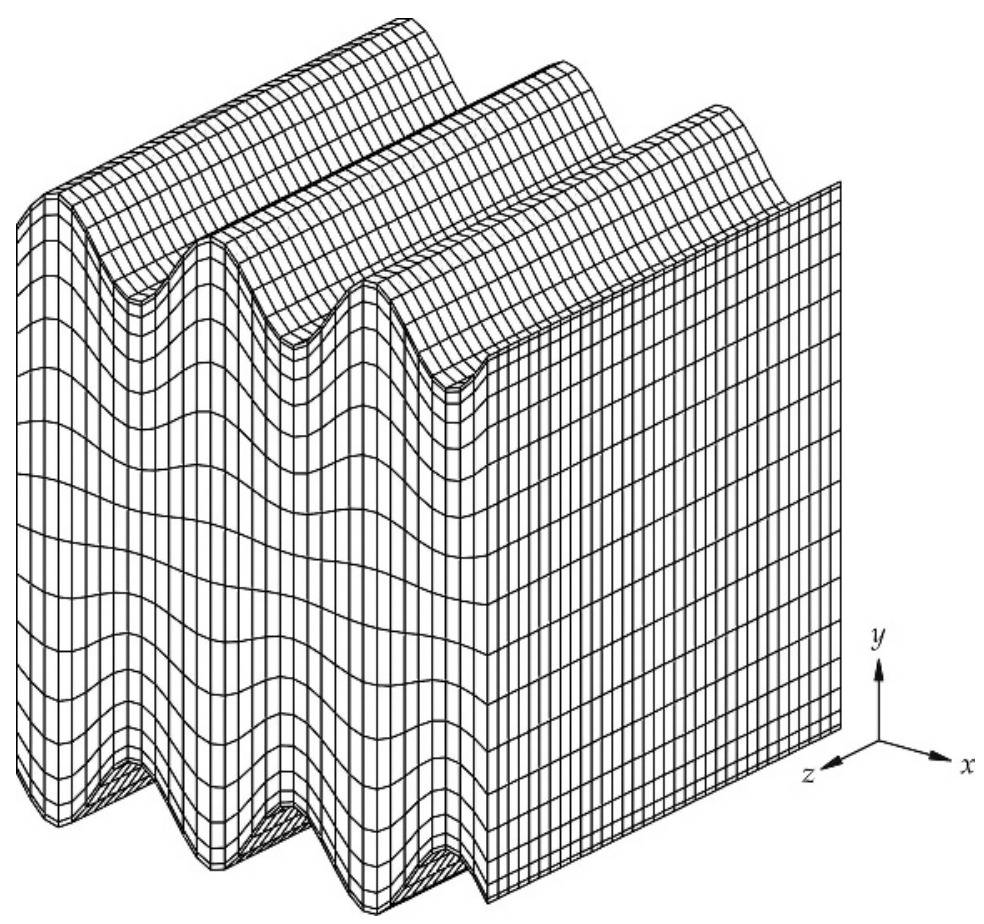

Figure 1.5 Distribution of displacements for the fundamental symmetric mode of Lamb waves

The notions of wavenumber $k$ and wavelength $\lambda$ are common for waves of every type. Wavenumber $k$ refers to the spatial frequency of perturbations. Wavelength $\lambda$ refers to the spatial period of perturbations (Figure 1.7) and is expressed by the following formula:

$$
\lambda=\frac{2 \pi}{k}
$$

Solution of Equation (1.1) can be expressed in a general form as:

$$
u(x, t)=\frac{U_{0}}{2}[\cos (k x-\omega t)+\cos (k x+\omega t)]
$$

where $U_{0}$ is wave amplitude and $\omega$ is angular velocity. The first term in square brackets is associated with wave propagation to the right (or 


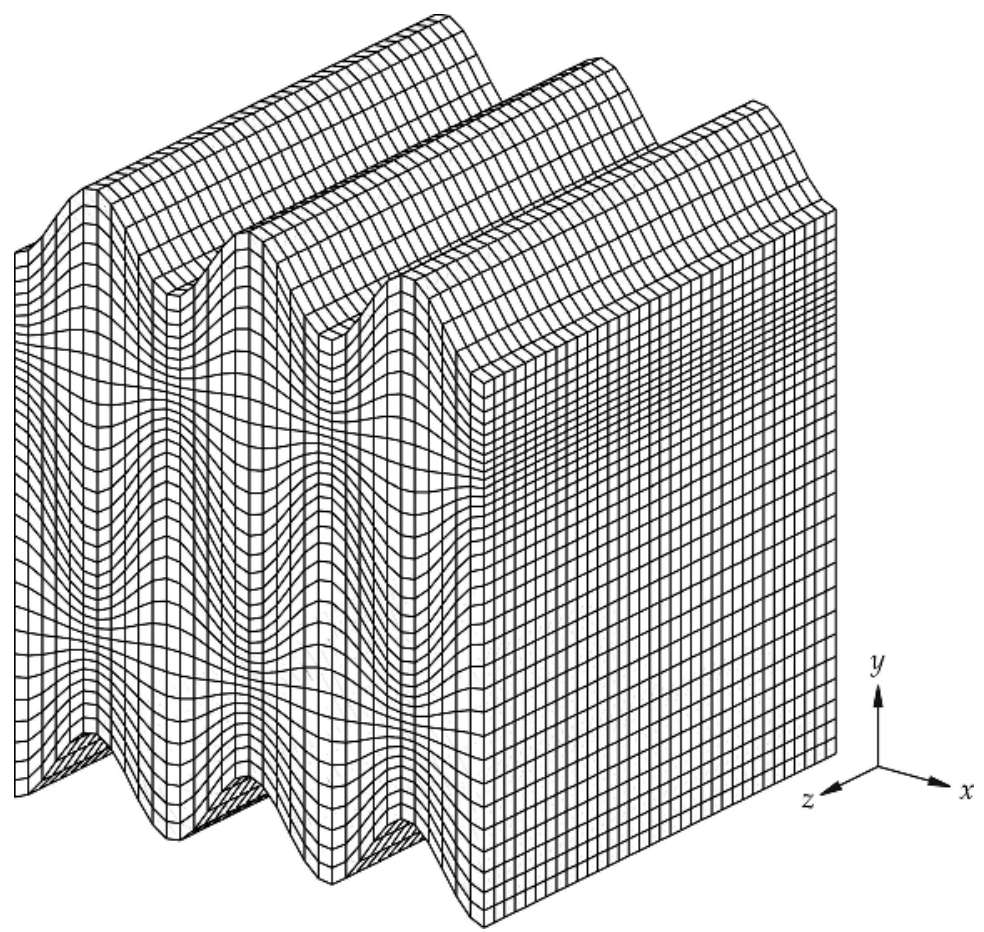

Figure 1.6 Distribution of displacements for the fundamental antisymmetric mode of Lamb waves

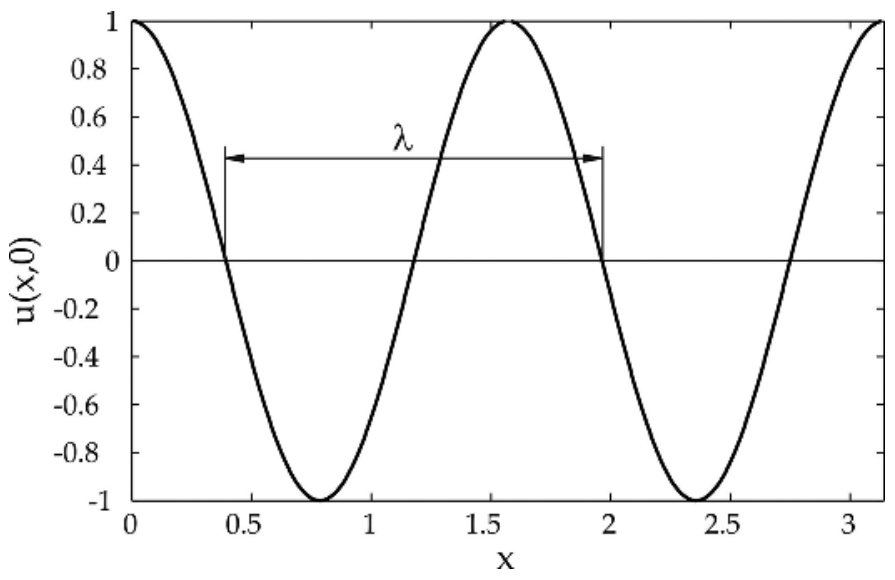

Figure 1.7 Harmonic wave of length $\lambda$ 


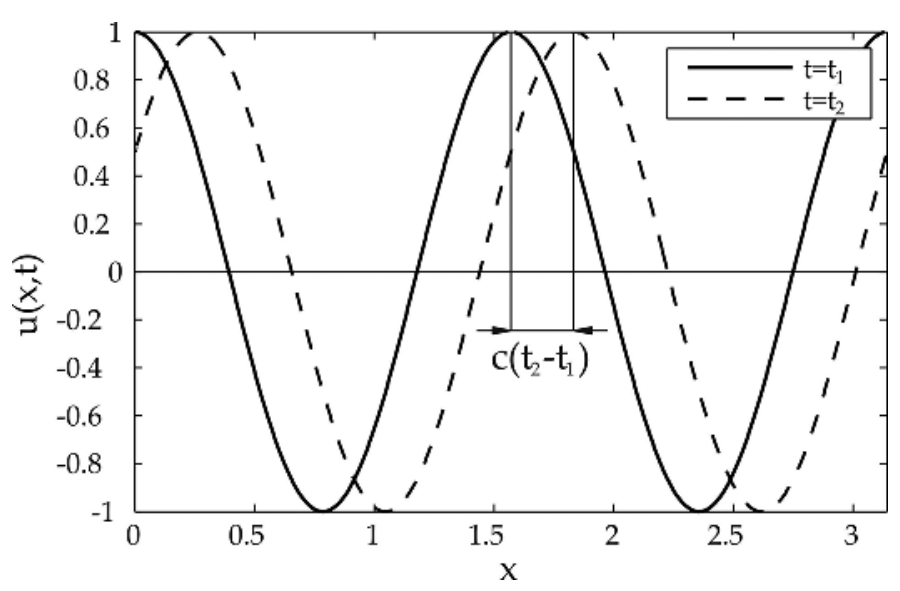

Figure 1.8 Harmonic wave propagating with velocity $c$

forwards), while the second term is associated with wave propagation to the left (or backwards). Considering a wave propagating to the right, this can be written as:

$$
u^{R}(x, t)=\frac{U_{0}}{2} \cos (k x-\omega t)
$$

The phase of this wave is $\phi=k x-\omega t$. For the constant phase $k x-\omega t=$ const it is $x=(\omega / k) t+$ const. Thus, a point of constant phase moves with velocity:

$$
c=\frac{\omega}{k}
$$

The harmonic wave propagating to the right with velocity $c$ is presented in Figure 1.8.

The phase velocity of a wave describes the relationship between spatial frequency $k$ and temporal frequency $\omega$ of the propagating waves. The dependency $\omega=\omega(k)$ is called the dispersion relationship. If this relation is linear, that is $\omega=c k$, the wave is nondispersive. In a nondispersive medium, the phase velocity is constant for all velocities.

Besides phase velocity, the term of group velocity is also associated with wave propagation. Group velocity refers to propagation of a group of waves called a wave packet. In order to understand the term of wave group velocity two waves propagating to the right, having the same amplitudes, but different 


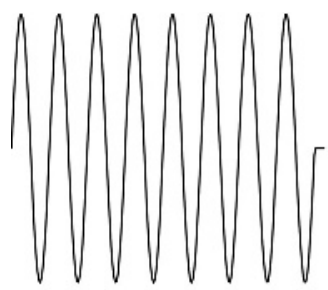

carrier wave

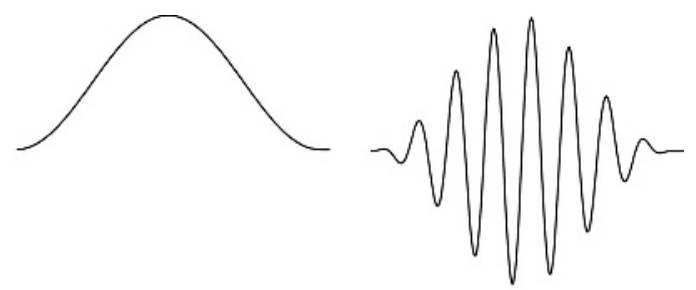

modulation wave wave packet

Figure 1.9 Wave packet as the superposition of a carrier wave and a modulating wave

frequencies and wavenumbers are considered:

$$
u(x, t)=U_{0}\left[\sin \left(k_{1} x-\omega_{1} t\right)+\sin \left(k_{2} x-\omega_{2} t\right)\right]
$$

Application of universally known trigonometric identities for the sum of sinus functions leads to:

$$
u(x, t)=2 U_{0} \cos \left(\frac{k_{1}-k_{2}}{2} x-\frac{\omega_{1}-\omega_{2}}{2} t\right) \sin \left(\frac{k_{1}+k_{2}}{2} x-\frac{\omega_{1}+\omega_{2}}{2} t\right)
$$

In formula (1.7) one can distinguish a term associated with modulation and one associated with a carrier wave:

$$
u(x, t)=2 U_{0} \underbrace{\cos (\Delta k x-\Delta \omega t)}_{\text {modulation }} \underbrace{\sin \left(k_{0} x-\omega_{0} t\right)}_{\text {carrier wave }}
$$

The wave packet is a superposition of a carrier wave and a modulating wave in the form of a window, as presented in Figure 1.9.

The propagation velocity of a modulating wave defines the propagation velocity of a wave packet. For a constant phase $\Delta k x-\Delta \omega t=$ const, this is $x=(\Delta \omega / \Delta k) t+$ const. Thus, group velocity at the limit transition $\Delta \omega \rightarrow 0$, $\Delta k \rightarrow 0$ is defined as:

$$
c_{g}=\frac{\mathrm{d} \omega}{\mathrm{d} k}
$$

One should note that for the nondispersive media the group velocity is equal to the phase velocity. In the dispersive media these velocities differ, which 
manifests directly as wave packet deformation during propagation. First of all, the wave packet amplitude decreases and the packet stretches.

\subsection{Bulk Waves in Three-Dimensional Media}

\subsection{Isotropic Media}

In infinite elastic medium waves propagate freely in every direction and are called bulk waves. The basis for discussing bulk waves is the threedimensional theory of elasticity. The full set of equations is as follows:

$$
\begin{aligned}
\sigma_{i j, j}+\rho f_{i} & =\rho \ddot{u}_{i}, \quad i, j=1,2,3 \\
\varepsilon_{i j} & =\frac{1}{2}\left(u_{i, j}+u_{j, i}\right), \quad i, j=1,2,3 \\
\sigma_{i j} & =\lambda \delta_{i j} \varepsilon_{k k}+2 \mu \varepsilon_{i j}, \quad i, j=1,2,3
\end{aligned}
$$

where $\varepsilon_{k k}=\varepsilon_{11}+\varepsilon_{22}+\varepsilon_{33}$ (Einstein summation convention) and $\delta_{i j}$ is the Kronecker delta. Equation (1.10) covers three motion equations, Equation (1.11) describes linear relationships between deformations and displacements (six independent equations) and Equation (1.12) covers six independent constitutive equations for the isotropic case. In Equation (1.12) Lame constants have been used; these are defined as:

$$
\begin{aligned}
\lambda & =\frac{v E}{(1+v)(1-2 v)} \\
\mu & =G=\frac{E}{2(1+v)}
\end{aligned}
$$

Equations (1.10) to (1.12) may be expanded using Cartesian notation. Thus, the motion equation can be written as:

$$
\begin{aligned}
& \frac{\partial \sigma_{x x}}{\partial x}+\frac{\partial \sigma_{x y}}{\partial y}+\frac{\partial \sigma_{x z}}{\partial z}+\rho f_{x}=\rho \frac{\partial^{2} u_{x}}{\partial t^{2}} \\
& \frac{\partial \sigma_{y x}}{\partial x}+\frac{\partial \sigma_{y y}}{\partial y}+\frac{\partial \sigma_{y z}}{\partial z}+\rho f_{y}=\rho \frac{\partial^{2} u_{y}}{\partial t^{2}} \\
& \frac{\partial \sigma_{z x}}{\partial x}+\frac{\partial \sigma_{z y}}{\partial y}+\frac{\partial \sigma_{z z}}{\partial z}+\rho f_{z}=\rho \frac{\partial^{2} u_{z}}{\partial t^{2}}
\end{aligned}
$$


where $\rho$ is the mass density. The relationships between stress components are governed by symmetry, that is $\sigma_{y x}=\sigma_{x y}, \sigma_{z y}=\sigma_{y z}, \sigma_{x z}=\sigma_{z x}$. The deformation-displacement equations take the following form:

$$
\begin{aligned}
\varepsilon_{x x} & =\frac{\partial u_{x}}{\partial x}, & \varepsilon_{x y} & =\frac{1}{2}\left(\frac{\partial u_{x}}{\partial y}+\frac{\partial u_{y}}{\partial x}\right) \\
\varepsilon_{y y} & =\frac{\partial u_{y}}{\partial y}, & \varepsilon_{y z} & =\frac{1}{2}\left(\frac{\partial u_{y}}{\partial z}+\frac{\partial u_{z}}{\partial y}\right) \\
\varepsilon_{z z} & =\frac{\partial u_{z}}{\partial z}, & \varepsilon_{z x} & =\frac{1}{2}\left(\frac{\partial u_{z}}{\partial x}+\frac{\partial u_{x}}{\partial z}\right)
\end{aligned}
$$

They are also subject to symmetry, that is $\varepsilon_{y x}=\varepsilon_{x y}, \varepsilon_{z y}=\varepsilon_{y z}, \varepsilon_{x z}=\varepsilon_{z x}$. The constitutive Equation (1.10) in Cartesian notation is as follows:

$$
\begin{aligned}
\sigma_{x x} & =(\lambda+2 \mu) \varepsilon_{x x}+\lambda \varepsilon_{y y}+\lambda \varepsilon_{z z}, & \sigma_{x y} & =2 \mu \varepsilon_{x y} \\
\sigma_{y y} & =\lambda \varepsilon_{x x}+(\lambda+2 \mu) \varepsilon_{y y}+\lambda \varepsilon_{z z}, & \sigma_{y z} & =2 \mu \varepsilon_{y z} \\
\sigma_{z z} & =\lambda \varepsilon_{x x}+\lambda \varepsilon_{y y}+(\lambda+2 \mu) \varepsilon_{z z}, & \sigma_{z x} & =2 \mu \varepsilon_{z x}
\end{aligned}
$$

Equations (1.14) and (1.15) remain valid for any continuous medium; the specific type of the discussed medium is introduced by Equations (1.16) in this case it is isotropic. Elimination of stresses and deformations from Equations (1.14) to (1.16) leads to:

$$
(\lambda+\mu) u_{j, j i}+\mu u_{i, j j}+\rho f_{i}=\rho \ddot{u}_{i}
$$

Motion Equations (1.17) containing only particle displacements are displacement-type partial differential equations. These equations are also known as Navier equations and in Cartesian notation take the following form [3]:

$$
\begin{aligned}
& (\lambda+\mu) \frac{\partial}{\partial x}\left(\frac{\partial u_{x}}{\partial x}+\frac{\partial u_{y}}{\partial y}+\frac{\partial u_{z}}{\partial z}\right)+\mu\left(\frac{\partial^{2} u_{x}}{\partial x^{2}}+\frac{\partial^{2} u_{x}}{\partial y^{2}}+\frac{\partial^{2} u_{x}}{\partial z^{2}}\right)+\rho f_{x}=\rho \frac{\partial^{2} u_{x}}{\partial t^{2}} \\
& (\lambda+\mu) \frac{\partial}{\partial y}\left(\frac{\partial u_{x}}{\partial x}+\frac{\partial u_{y}}{\partial y}+\frac{\partial u_{z}}{\partial z}\right)+\mu\left(\frac{\partial^{2} u_{y}}{\partial x^{2}}+\frac{\partial^{2} u_{y}}{\partial y^{2}}+\frac{\partial^{2} u_{y}}{\partial z^{2}}\right)+\rho f_{y}=\rho \frac{\partial^{2} u_{y}}{\partial t^{2}} \\
& (\lambda+\mu) \frac{\partial}{\partial z}\left(\frac{\partial u_{x}}{\partial x}+\frac{\partial u_{y}}{\partial y}+\frac{\partial u_{z}}{\partial z}\right)+\mu\left(\frac{\partial^{2} u_{z}}{\partial x^{2}}+\frac{\partial^{2} u_{z}}{\partial y^{2}}+\frac{\partial^{2} u_{z}}{\partial z^{2}}\right)+\rho f_{z}=\rho \frac{\partial^{2} u_{z}}{\partial t^{2}}
\end{aligned}
$$


If the area where the solution is sought is infinite, these equations are sufficient for describing elastic wave propagation. If the area is finite, on the other hand, boundary conditions are necessary for the problem to be well-posed. These boundary conditions take the form of imposed stresses and/or displacements at area boundaries.

\subsubsection{Christoffel Equations for Anisotropic Media}

Wave propagation in infinite anisotropic elastic solids is governed by the full set of equations of the three-dimensional theory of elasticity. Compared to the isotropic case, the difference lies in a more general constitutive equation. The full set of equations of the theory of elasticity for homogeneous anisotropic media is as follows:

$$
\begin{aligned}
\sigma_{i k, k}+\rho f_{i} & =\rho \ddot{u}_{i}, \quad i, k=1,2,3 \\
\varepsilon_{l m} & =\frac{1}{2}\left(u_{l, m}+u_{m, l}\right), \quad l, m=1,2,3 \\
\sigma_{i k} & =C_{i k l m} \varepsilon_{l m}, \quad i, k, l, m=1,2,3
\end{aligned}
$$

By combining Equations (1.19), (1.20) and (1.21) and ignoring external forces the motion equations are obtained:

$$
\frac{1}{2} C_{i k l m}\left(u_{l, k m}+u_{m, k l}\right)=\rho \ddot{u}_{i}
$$

The tensor of elasticity constants $C_{i k l m}$ is symmetric with regard to $l$ and $m$, and therefore:

$$
C_{i k l m}=C_{i k m l}=C_{k i m}
$$

A flat harmonic plane wave propagating forwards is assumed:

$$
u_{\mathrm{i}}=A_{\mathrm{i}} \mathrm{e}^{\mathrm{i}\left(k_{j} x_{j}-\omega t\right)}
$$

where $\mathrm{i}=\sqrt{-1}$ is the imaginary unit, $k_{j}$ is the wavenumber, $A_{\mathrm{i}}$ is a vector of wave amplitudes and $\omega$ is angular frequency. Substitution of Equation (1.24) into Equation (1.22) leads to:

$$
C_{i k l m} k_{k} k_{l} u_{m}=\rho \omega^{2} u_{i}
$$


It can be seen that $u_{i}=u_{m} \delta_{i m}$; therefore:

$$
\left(\rho \omega^{2} \delta_{i m}-C_{i k l m} k_{k} k_{l}\right) u_{m}=0
$$

This is the Christoffel equation for an anisotropic medium. The Christoffel tensor can be defined as:

$$
\lambda_{i m}=\Gamma_{i m}=C_{i k l m} n_{k} n_{l}
$$

where $n_{k}$ are direction cosines normal to the wavefront. Furthermore, taking into account the relationships:

$$
k_{k}=k n_{k}, \quad k_{l}=k n_{l}
$$

leads to:

$$
\left(\Gamma_{i m} k^{2}-\rho \omega^{2} \delta_{i m}\right) u_{m}=0
$$

By recalling the definition of phase velocity:

$$
c=\frac{\omega}{k}
$$

Equation (1.29) is brought to the following form:

$$
\left(\Gamma_{i m}-\rho c^{2} \delta_{i m}\right) u_{m}=0
$$

This is a uniform system of three equations. The system has a nontrivial solution if the determinant of the coefficient matrix is equal to zero. This is a classic eigenvalue problem. The solution is composed of three velocities (eigenvalues with regard to $c^{2}$ ) and the corresponding eigenvectors. Depending on the arrangement of eigenvectors in space, one can be dealing with: a P wave together with $\mathrm{SH}$ and SV waves, a quasi-P wave together with $\mathrm{SH}$ and SV waves, a P wave together with quasi-SH and quasi-SV waves or a quasi$\mathrm{P}$ wave together with quasi-SH and quasi-SV waves [4]. One should note that phase velocities depend on the direction of propagation, which results from the definition of the Christoffel tensor (Equation (1.27)). In an isotropic medium there are always pure waves: a longitudinal one and two shear ones, the phase velocities of which do not depend on the direction of propagation. 


\subsubsection{Potential Method}

Bulk waves connected with wave propagation in an isotropic infinite media are considered in this section. When no external forces $f$ are present, Equation (1.18) can be expressed in vector form as:

$$
(\lambda+\mu) \nabla(\nabla \cdot u)+\mu \nabla^{2} \boldsymbol{u}=\rho \ddot{\boldsymbol{u}}
$$

The motion Equation (1.32) can be simplified further by applying Helmholtz decomposition and the potential method [4-6]. Such an operation is only possible for isotropic media. It is assumed that the displacement vector $u$ can be expressed through two potential functions: the scalar potential $\Phi$ and the vector potential $\boldsymbol{H}=H_{x} \boldsymbol{i}+H_{y} \boldsymbol{j}+H_{z} \boldsymbol{k}$, that is:

$$
\boldsymbol{u}=\nabla \Phi+\nabla \times \boldsymbol{H}
$$

Equation (1.33) is known as the Helmholtz solution complemented by the condition:

$$
\nabla \cdot H=0
$$

By applying Equation (1.33), components of Equation (1.32) can be expressed as:

$$
\begin{aligned}
\nabla \cdot \boldsymbol{u} & =\nabla(\nabla \Phi+\nabla \times \boldsymbol{H})=(\nabla \cdot \nabla) \Phi+\underbrace{\nabla \cdot(\nabla \times \boldsymbol{H})}_{=0}=\nabla^{2} \Phi \\
\nabla^{2} \boldsymbol{u} & =\nabla^{2}(\nabla \Phi+\nabla \times \boldsymbol{H})=\nabla^{2} \nabla \Phi+\nabla^{2} \nabla \times \boldsymbol{H} \\
\ddot{\boldsymbol{u}} & =\nabla \ddot{\Phi}+\nabla \times \ddot{\boldsymbol{H}}
\end{aligned}
$$

By substituting Equations (1.35), (1.36) and (1.37) into Equation (1.32) the following formula is obtained:

$$
(\lambda+\mu) \nabla\left(\nabla^{2} \Phi\right)+\mu\left(\nabla^{2} \nabla \Phi+\nabla^{2} \nabla \times \boldsymbol{H}\right)=\rho(\nabla \ddot{\Phi}+\nabla \times \ddot{H})
$$

Noting that $\nabla \nabla^{2}=\nabla^{2} \nabla$ (commutativity of differentiation), Equation (1.38) after transformations yields:

$$
\nabla\left((\lambda+2 \mu) \nabla^{2} \Phi-\rho \ddot{\Phi}\right)+\nabla \times\left(\mu \nabla^{2} \boldsymbol{H}-\rho \ddot{\boldsymbol{H}}\right)=\mathbf{0}
$$


Equation (1.39) is satisfied for any point in space at any time, if the terms in parentheses vanish, that is:

$$
\begin{aligned}
& (\lambda+2 \mu) \nabla^{2} \Phi-\rho \ddot{\Phi}=0 \\
& \mu \nabla^{2} \boldsymbol{H}-\rho \ddot{\boldsymbol{H}}=\mathbf{0}
\end{aligned}
$$

After dividing by $\rho$ and ordering, Equations (1.40) and (1.41) become wave equations for the scalar potential $\Phi$ and the vector potential $H$, that is:

$$
\begin{aligned}
c_{L} \nabla^{2} \Phi & =\ddot{\Phi} \\
c_{S} \nabla^{2} \boldsymbol{H} & =\ddot{\boldsymbol{H}}
\end{aligned}
$$

where $c_{L}$ is the longitudinal wave velocity, defined as:

$$
c_{L}=\sqrt{\frac{\lambda+2 \mu}{\rho}}
$$

and $c_{S}$ is the shear wave velocity, defined as:

$$
c_{S}=\sqrt{\frac{\mu}{\rho}}
$$

As a result, the motion Equation (1.32) was decomposed into two simplified wave Equations (1.42) and (1.43). Assuming that the rotational part $\nabla \times \boldsymbol{H}$ of Equation (1.33) is equal to zero, the longitudinal wave equation is obtained:

$$
c_{L} \nabla^{2} u=\ddot{u}
$$

Assuming that displacements in Equation (1.33) contain the rotational part only, the shear wave equation is obtained as:

$$
c_{S} \nabla^{2} u=\ddot{u}
$$

\subsection{Plane Waves}

A specific case of three-dimensional waves are plane waves. These waves are invariant in one direction along the wave crest. Such a situation happens 
when the wave crest is parallel to the $z$ axis (cf. Figures 1.1 to 1.6). Moreover, the normal vector of the wave crest is perpendicular to the $z$ axis. Invariance in the direction of the $z$ axis means that all wave functions are independent of $z$, and therefore their derivatives with respect to $z$ are equal to zero, that is:

$$
\frac{\partial}{\partial z} \equiv 0 \quad \text { and } \quad \nabla=i \frac{\partial}{\partial x}+j \frac{\partial}{\partial y}
$$

After substituting Equation (1.48) into Equation (1.33) and expanding, the expression for displacement is obtained:

$$
\boldsymbol{u}=\underbrace{\left(\frac{\partial \Phi}{\partial x}+\frac{\partial H_{z}}{\partial y}\right)}_{u_{x}} \boldsymbol{i}+\underbrace{\left(\frac{\partial \Phi}{\partial y}-\frac{\partial H_{z}}{\partial x}\right)}_{u_{y}} \boldsymbol{j}+\underbrace{\left(\frac{\partial H_{y}}{\partial x}-\frac{\partial H_{x}}{\partial y}\right)}_{u_{z}} \boldsymbol{k}
$$

Although movement is invariant with respect to the $z$ axis, Equation (1.49) indicates that displacement components appear in all three directions $(x, y$ and $z$ ). It is noteworthy that the displacement component $u_{z}$ depends only on potentials $H_{x}$ and $H_{y}$ that are associated with the horizontally polarised shear wave (SH wave). Displacement components $u_{x}$ and $u_{y}$ depend on potentials $\Phi$ and $H_{z}$ associated with the longitudinal wave (P wave) and vertically polarised shear wave (SV wave), respectively. Thanks to these relations one can seek solutions of wave equations separately for the $\mathrm{SH}$ wave and the $\mathrm{P}+\mathrm{SV}$ wave combination.

\subsubsection{Surface Waves}

An example of waves propagating near a solid surface are Rayleigh waves, the amplitude of which decreases rapidly with depth. The effective penetration depth for Rayleigh waves is smaller than their wavelength. Particle movement takes place in the vertical plane and is independent of the $z$ direction; therefore one can seek solutions in terms of P + SV type plane waves. The following assumptions are made:

$$
\frac{\partial}{\partial z}=0, \quad u_{x} \neq 0, \quad u_{y} \neq 0, \quad u_{z}=0
$$

The wave Equations (1.42) and (1.43) can then be expressed in terms of potentials $\Phi$ and $H_{z}$ :

$$
c_{L} \nabla^{2} \Phi=\ddot{\Phi}, \quad c_{S} \nabla^{2} H_{z}=\ddot{H}_{z}
$$


Assuming potentials of the following forms:

$$
\Phi(x, y, t)=f(y) \mathrm{e}^{\mathrm{i}(k x-\omega t)}, \quad H_{z}(x, y, t)=h_{z}(y) \mathrm{e}^{\mathrm{i}(k x-\omega t)}
$$

where $k$ denotes the wavenumber, and then imposing boundary conditions (stresses vanishing on the bounding surface):

$$
\left.\sigma_{y y}\right|_{y=0}=0,\left.\quad \sigma_{x y}\right|_{y=0}=0
$$

After transformations the characteristic equation is obtained:

$$
\left(\beta^{2}+k^{2}\right)^{2}-4 \alpha \beta k^{2}=0
$$

where $\alpha$ and $\beta$ depend on the frequency $\omega$ and the wavenumber $k$ :

$$
\alpha^{2}=k^{2}-\frac{\omega^{2}}{c_{L}^{2}}, \quad \beta^{2}=k^{2}-\frac{\omega^{2}}{c_{S}^{2}}
$$

Even though Equation (1.54) has three double roots, only one of them is real. The real root corresponds to the surface Rayleigh wave velocity: $c_{R}=\omega / k_{R}$. By transforming Equation (1.54) one can show that the Rayleigh wave velocity depends on the Poisson coefficient $v$ and the shear wave velocity $c_{S}$ [5]. A universally accepted Rayleigh wave velocity approximation formula is:

$$
c_{R}(v)=c_{S}\left(\frac{0.87+1.12 v}{1+v}\right)
$$

Particle movement for Rayleigh waves can be described as follows:

$$
\begin{aligned}
& u_{x}(y)=A \mathrm{i}\left(k \mathrm{e}^{-\alpha y}-\frac{\beta^{2}+k^{2}}{2 k} \mathrm{e}^{-\beta y}\right) \\
& u_{y}(y)=A\left(-\alpha \mathrm{e}^{-\alpha y}+\mathrm{i} \frac{\beta^{2}+k^{2}}{2 \beta} \mathrm{e}^{-\beta y}\right)
\end{aligned}
$$

where $A$ is any constant.

\subsubsection{Derivation of Lamb Wave Equations}

The P + SV wave combination that leads to Lamb wave equations is considered in this section. Lamb wave propagation in a free plate of shape as 


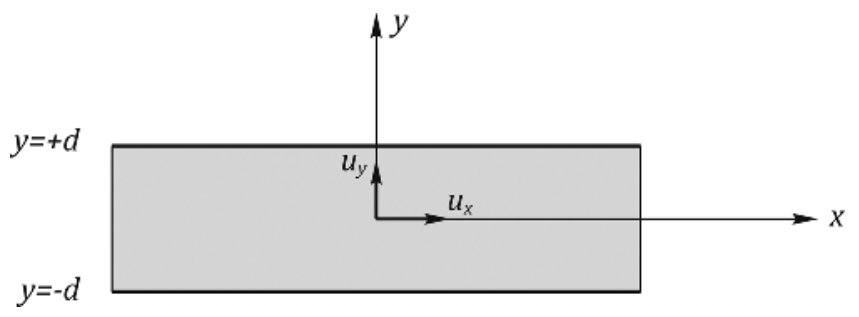

Figure 1.10 Shape of the free plate in the Lamb wave problem

presented in Figure 1.10 is investigated. The problem is described by the motion Equations (1.42) and (1.43) with boundary conditions imposed on the plate surfaces. It is assumed that no stress is present on surfaces of coordinates $y=d$ and $y=-d$. Exciting the plate at any point causes the propagating excited impulse to encounter the top and bottom surfaces of the plate edge. By means of multiple reflections from the top and bottom plate surfaces as well as constructive and destructive interference of $\mathrm{P}$ and SV waves, Lamb waves are generated. Lamb waves are composed of waves standing in the thickness direction $y$ (Lamb wave modes) and propagating in the $x$ direction.

In the case of the solution applicable to the P + SV wave combination, motion is contained in the vertical $(x, y)$ plane and the following conditions are observed:

$$
u_{x} \neq 0, \quad u_{y} \neq 0, \quad \frac{\partial}{\partial z}=0, \quad \Phi \text { and } H_{z} \text { only }
$$

In order to simplify the notation, the two potential functions $\Phi$ and $H_{z}$ are denoted as $\phi$ and $\psi$, respectively. Substituting conditions (1.58) into Equations (1.42) and (1.43) the following expressions are obtained:

$$
\begin{array}{ll}
\frac{\partial^{2} \phi}{\partial x^{2}}+\frac{\partial^{2} \phi}{\partial y^{2}}=\frac{1}{c_{L}^{2}} \frac{\partial^{2} \phi}{\partial t^{2}} & \text { longitudinal waves } \\
\frac{\partial^{2} \psi}{\partial x^{2}}+\frac{\partial^{2} \psi}{\partial y^{2}}=\frac{1}{c_{S}^{2}} \frac{\partial^{2} \psi}{\partial t^{2}} & \text { shear waves }
\end{array}
$$

Referring to the displacement field expressed through potentials (1.49):

$$
u_{x}=\frac{\partial \phi}{\partial x}+\frac{\partial \psi}{\partial y}, \quad u_{y}=\frac{\partial \phi}{\partial y}-\frac{\partial \psi}{\partial x}, \quad u_{z}=0
$$


and substituting Equations (1.60) into the deformation-displacement relationship (1.15), the strain relations are obtained as:

$$
\begin{aligned}
\varepsilon_{x x} & =\frac{\partial^{2} \phi}{\partial x^{2}}+\frac{\partial^{2} \psi}{\partial x \partial y} \\
\varepsilon_{y y} & =\frac{\partial^{2} \phi}{\partial y^{2}}-\frac{\partial^{2} \psi}{\partial x \partial y} \\
\varepsilon_{y x} & =\frac{1}{2}\left(2 \frac{\partial^{2} \phi}{\partial x \partial y}-\frac{\partial^{2} \psi}{\partial x^{2}}+\frac{\partial^{2} \psi}{\partial y^{2}}\right) \\
\varepsilon_{z z} & =0 \\
\varepsilon_{y z} & =0 \\
\varepsilon_{z x} & =0
\end{aligned}
$$

Stress, in turn, can be expressed according to Equations (1.16) as:

$$
\begin{aligned}
& \sigma_{x x}=\lambda\left(\frac{\partial^{2} \phi}{\partial x^{2}}+\frac{\partial^{2} \phi}{\partial y^{2}}\right)+2 \mu\left(\frac{\partial^{2} \phi}{\partial x^{2}}+\frac{\partial^{2} \psi}{\partial x \partial y}\right) \\
& \sigma_{y y}=\lambda\left(\frac{\partial^{2} \phi}{\partial x^{2}}+\frac{\partial^{2} \phi}{\partial y^{2}}\right)+2 \mu\left(\frac{\partial^{2} \phi}{\partial y^{2}}-\frac{\partial^{2} \psi}{\partial x \partial y}\right) \\
& \sigma_{z z}=0 \\
& \sigma_{y x}=\mu\left(2 \frac{\partial^{2} \phi}{\partial x \partial y}-\frac{\partial^{2} \psi}{\partial x^{2}}+\frac{\partial^{2} \psi}{\partial y^{2}}\right) \\
& \sigma_{y z}=0 \\
& \sigma_{z x}=0
\end{aligned}
$$

The solution of Equations (1.59) is assumed in the following form:

$$
\begin{aligned}
& \phi=\Phi(y) \mathrm{e}^{\mathrm{i}(k x-\omega t)} \\
& \psi=\Psi(y) \mathrm{e}^{\mathrm{i}(k x-\omega t)}
\end{aligned}
$$

It should be noticed that these solutions represent waves propagating in the $x$ direction and waves standing in the $y$ direction. The complex term of the exponential function includes a time variable depending on $x$, which is associated with wave propagation. On the other hand, the unknown functions $\Phi$ and $\Psi$ are 'static' functions that only depend on $y$. In other words, these functions describe the stress distribution in the crosswise direction (across the plate thickness). Substituting the relationships (1.63) into Equations (1.59) 
leads to a system of differential equations with regard to functions $\Phi$ and $\Psi$ :

$$
\begin{aligned}
& \frac{\partial^{2} \Phi}{\partial y^{2}}+\left(\frac{\omega^{2}}{c_{L}^{2}}-k^{2}\right) \Phi=0 \\
& \frac{\partial^{2} \Psi}{\partial y^{2}}+\left(\frac{\omega^{2}}{c_{S}^{2}}-k^{2}\right) \Psi=0
\end{aligned}
$$

In the same fashion displacements and stress can be directly obtained from Equations (1.60) and (1.62). By ignoring the $\mathrm{e}^{\mathrm{i}(k x-\omega t)}$ term in all expressions, the displacements and stresses can be expressed as:

$$
\begin{aligned}
u_{x} & =\mathrm{i} k \Phi+\frac{\mathrm{d} \Psi}{\mathrm{d} y}, \quad u_{y}=\frac{\mathrm{d} \Phi}{\mathrm{d} y}-\mathrm{i} k \Psi \\
\sigma_{x x} & =\lambda\left(-k^{2} \Phi+\frac{\mathrm{d}^{2} \Phi}{\mathrm{d} y^{2}}\right)+2 \mu\left(-k^{2} \Phi+\mathrm{i} k \frac{\mathrm{d} \Psi}{\mathrm{d} y}\right) \\
\sigma_{y y} & =\lambda\left(-k^{2} \Phi+\frac{\mathrm{d}^{2} \Phi}{\mathrm{d} y^{2}}\right)+2 \mu\left(\frac{\mathrm{d}^{2} \Phi}{\mathrm{d} y^{2}}-\mathrm{i} k \frac{\mathrm{d} \Psi}{\mathrm{d} y}\right) \\
\sigma_{y x} & =\mu\left(2 \mathrm{i} k \frac{\mathrm{d} \Phi}{\mathrm{d} y}+k^{2} \Psi+\frac{\mathrm{d}^{2} \Psi}{\mathrm{d} y^{2}}\right)
\end{aligned}
$$

After the following symbols are introduced:

$$
p^{2}=\frac{\omega^{2}}{c_{L}^{2}}-k^{2}, \quad q^{2}=\frac{\omega^{2}}{c_{S}^{2}}-k^{2}
$$

Equations (1.64) are brought to the following form:

$$
\begin{aligned}
& \frac{\partial^{2} \Phi}{\partial y^{2}}+p^{2} \Phi=0 \\
& \frac{\partial^{2} \Psi}{\partial y^{2}}+q^{2} \Psi=0
\end{aligned}
$$

Equations (1.67) are fulfilled by a general solution:

$$
\begin{aligned}
& \Phi=A_{1} \sin (p y)+A_{2} \cos (p y) \\
& \Psi=B_{1} \sin (q y)+B_{2} \cos (q y)
\end{aligned}
$$


Derivatives of potentials with regard to $y$ are as follows:

$$
\begin{array}{ll}
\frac{\mathrm{d} \Phi}{\mathrm{d} y}=A_{1} p \cos (p y)-A_{2} \sin (p y), & \frac{\mathrm{d}^{2} \Phi}{\mathrm{d} y^{2}}=-A_{1} p^{2} \sin (p y)-A_{2} p^{2} \cos (p y) \\
\frac{\mathrm{d} \Psi}{\mathrm{d} y}=B_{1} q \cos (q y)-B_{2} \sin (q y), & \frac{\mathrm{d}^{2} \Psi}{\mathrm{d} y^{2}}=-B_{1} q^{2} \sin (q y)-B_{2} q^{2} \cos (q y)
\end{array}
$$

As field variables contain sine and cosine functions with argument $y$, which are odd and even with regard to $y=0$, respectively, the solution can be sorted into two sets of modes: symmetric modes and antisymmetric ones. Specifically, the distribution of displacements in the direction of the $x$ axis will be symmetric with respect to the middle plane of the plate when $u_{x}$ contains cosines and antisymmetric when $u_{x}$ contains sines. This is reversed for displacements in the direction of the $y$ axis. Thus, equation systems for individual wave propagation modes are as follows.

\subsubsection{Symmetric Modes}

$$
\begin{aligned}
\Phi^{S} & =A_{2} \cos (p y) \\
\Psi^{S} & =B_{1} \sin (p y) \\
u_{x}^{S} & =A_{2} \mathrm{i} k \cos (p y)+B_{1} q \cos (q y) \\
u_{y}^{S} & =-A_{2} p \sin (p y)+B_{1} \mathrm{i} k \sin (q y) \\
\sigma_{x x}^{S} & =-A_{2}\left(\lambda p^{2}+(\lambda+2 \mu) k^{2}\right) \cos (p y)-B_{1} 2 \mu \mathrm{i} k q \cos (q y) \\
\sigma_{y y}^{S} & =-A_{2}\left(\lambda k^{2}+(\lambda+2 \mu) p^{2}\right) \cos (p y)-B_{1} 2 \mu \mathrm{i} k q \cos (q y) \\
\sigma_{y x}^{S} & =\mu\left[-A_{2} 2 \mathrm{i} k p \sin (p y)+B_{1}\left(k^{2}-q^{2}\right) \sin (q y)\right]
\end{aligned}
$$

\subsubsection{Antisymmetric Modes}

$$
\begin{aligned}
\Phi^{A} & =A_{1} \sin (p y) \\
\Psi^{A} & =B_{2} \cos (p y) \\
u_{x}^{A} & =A_{1} \mathrm{i} k \sin (p y)-B_{2} q \sin (q y) \\
u_{y}^{A} & =A_{1} p \cos (p y)-B_{2} \mathrm{i} k \cos (q y) \\
\sigma_{x x}^{A} & =-A_{1}\left(\lambda p^{2}+(\lambda+2 \mu) k^{2}\right) \sin (p y)-B_{2} 2 \mu \mathrm{i} k q \sin (q y) \\
\sigma_{y y}^{A} & =-A_{1}\left(\lambda k^{2}+(\lambda+2 \mu) p^{2}\right) \sin (p y)+B_{2} 2 \mu \mathrm{i} k q \sin (q y) \\
\sigma_{y x}^{A} & =\mu\left[A_{1} 2 \mathrm{i} k p \cos (p y)+B_{2}\left(k^{2}-q^{2}\right) \cos (q y)\right]
\end{aligned}
$$


Using the relationship:

$$
\begin{aligned}
\lambda k^{2}+(\lambda+2 \mu) p^{2} & =\lambda k^{2}+(\lambda+2 \mu)\left(\frac{\omega^{2}}{c_{L}^{2}}-k^{2}\right) \\
& =\lambda k^{2}-k^{2}(\lambda+2 \mu)+(\lambda+2 \mu)\left(\frac{\omega^{2}}{c_{L}^{2}}\right) \\
& =-2 k^{2} \mu+\omega^{2} \rho=\mu\left(-2 k^{2}+\frac{\omega^{2}}{c_{S}^{2}}\right) \\
& =\mu\left[\left(\frac{\omega^{2}}{c_{S}^{2}}-k^{2}\right)-k^{2}\right]=\mu\left(q^{2}-k^{2}\right)
\end{aligned}
$$

gives:

$$
\begin{aligned}
& \sigma_{y y}^{S}=-A_{2} \mu\left(q^{2}-k^{2}\right) \cos (p y)-B_{1} 2 \mu \mathrm{i} k q \cos (q y) \\
& \sigma_{y y}^{A}=-A_{1} \mu\left(q^{2}-k^{2}\right) \sin (p y)+B_{2} 2 \mu \mathrm{i} k q \sin (q y)
\end{aligned}
$$

One should note that the waves can be separated into symmetric and antisymmetric modes only in specific cases, when structure symmetry is present. Such separation is impossible in the case of analysis of anisotropic plates, unless the wave propagates along the symmetry plane of the plate.

Constants $A_{1}, A_{2}, B_{1}, B_{2}$, as well as dispersion equations, still remain unknown. They can be obtained by imposing free boundary conditions.

\subsubsection{Symmetric Solution}

The symmetric solution of Lamb wave equations is obtained when displacements and stresses are assumed to be symmetrical with respect to the middle plane (see Figure 1.11):

$$
\begin{aligned}
& u_{x}(x,-d)=u_{x}(x, d), \quad \sigma_{y x}(x,-d)=-\sigma_{y x}(x, d) \\
& u_{y}(x,-d)=-u_{y}(x, d), \quad \sigma_{y y}(x,-d)=\sigma_{y y}(x, d)
\end{aligned}
$$

One should note that positive shear stresses have the same directions on the top and bottom surfaces, and thus the opposite signs in Equations (1.74). Symmetric boundary conditions are as follows:

$$
\begin{aligned}
& \sigma_{y x}(x,-d)=-\sigma_{y x}(x, d)=0 \\
& \sigma_{y y}(x,-d)=\sigma_{y y}(x, d)=0
\end{aligned}
$$



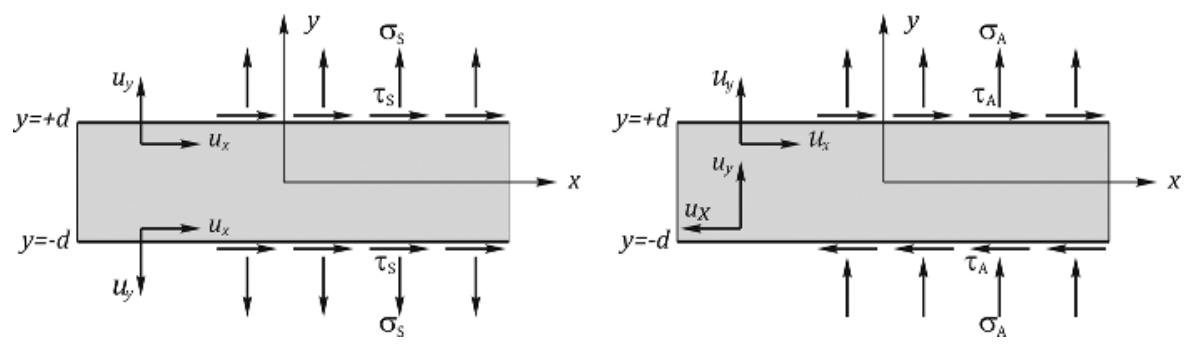

Figure 1.11 Symmetric and antisymmetric analysis

After substituting boundary conditions into the stress relationships described by formulas (1.70) and (1.73) a set of linear equations is obtained:

$$
\left[\begin{array}{rl}
-2 \mathrm{i} k p \sin (p d) & \left(k^{2}-q^{2}\right) \sin (q d) \\
\left(k^{2}-q^{2}\right) \cos (p d) & -2 \mathrm{i} k q \cos (q d)
\end{array}\right]\left[\begin{array}{l}
A_{2} \\
B_{1}
\end{array}\right]=\left[\begin{array}{l}
0 \\
0
\end{array}\right]
$$

A uniform system of linear equations can be solved when its determinant is equal to zero:

$$
D_{S}=\left(k^{2}-q^{2}\right)^{2} \cos (p d) \sin (q d)+4 k^{2} p q \sin (p d) \cos (q d)=0
$$

After transformations:

$$
\frac{\tan (q d)}{\tan (p d)}=-\frac{4 k^{2} p q}{\left(k^{2}-q^{2}\right)^{2}} \quad \text { symmetric modes }
$$

One should note that $p$ and $q$ depend on the wavenumber $k$ as well as on the frequency $f=\omega / 2 \pi$. Equation (1.78) is known as the Rayleigh-Lamb frequency equation or the dispersion equation. Using this equation, one can compute the symmetric mode (S) velocity, with which waves propagate in a plate of thickness $d$ for a chosen frequency $f$. A numerical solution of Equation (1.78) is a set of symmetric eigenvalues $k_{0}^{S}, k_{1}^{S}, k_{2}^{S}, \ldots$ Substituting the eigenvalues into the uniform system of Equations (1.76) allows thecoefficients $\left(A_{2}, B_{1}\right)$ to be determined in the form:

$$
A_{2}=2 \mathrm{i} k q \cos (q d), \quad B_{1}=\left(k^{2}-q^{2}\right) \cos (p d)
$$


Substitution of the coefficients $\left(A_{2}, B_{1}\right)$ into Equations (1.70) yields symmetric modes of the Lamb waves:

$$
\begin{aligned}
& u_{x}^{S}=-2 k^{2} q \cos (q d) \cos (p y)+q\left(k^{2}-q^{2}\right) \cos (p d) \cos (q y) \\
& u_{y}^{S}=-2 \mathrm{i} k p q \cos (q d) \sin (p y)-\mathrm{i} k\left(k^{2}-q^{2}\right) \cos (p d) \sin (q y)
\end{aligned}
$$

Stress distribution is obtained by means of substituting the coefficients $\left(A_{2}, B_{1}\right)$ into Equations (1.70) and (1.73):

$$
\begin{aligned}
\sigma_{x x}^{S}= & -(2 \mathrm{i} k q)\left[\left(\lambda p^{2}+(\lambda+2 \mu) k^{2}\right) \cos (q d) \cos (p y)\right. \\
& \left.+\mu\left(q^{2}-k^{2}\right) \cos (p d) \cos (q y)\right] \\
\sigma_{y y}^{S}= & -2 \mu(\mathrm{i} k q)\left(k^{2}-q^{2}\right)[\cos (q d) \cos (p y)-\cos (p d) \cos (q y)] \\
\sigma_{y x}^{S}= & \mu\left[4 k^{2} p q \cos (q d) \sin (p y)+\left(k^{2}-q^{2}\right)^{2} \cos (p d) \sin (q y)\right]
\end{aligned}
$$

\subsubsection{Antisymmetric Solution}

An antisymmetric solution of the Lamb wave equations is obtained when displacements and stresses are assumed to be antisymmetrical with respect to the middle plane (see Figure 1.11):

$$
\begin{array}{ll}
u_{x}(x,-d)=-u_{x}(x, d), & \sigma_{y x}(x,-d)=\sigma_{y x}(x, d) \\
u_{y}(x,-d)=u_{y}(x, d), & \sigma_{y y}(x,-d)=-\sigma_{y y}(x, d)
\end{array}
$$

One should note that positive shear stresses have opposite directions on the top and bottom surfaces, and thus they are antisymmetric. Antisymmetric boundary conditions are as follows:

$$
\begin{aligned}
& \sigma_{y x}(x,-d)=\sigma_{y x}(x, d)=0 \\
& \sigma_{y y}(x,-d)=-\sigma_{y y}(x, d)=0
\end{aligned}
$$

After substituting boundary conditions into the stress relationships expressed by formulas (1.71) and (1.73) a set of linear equations is obtained:

$$
\left[\begin{array}{cc}
2 \mathrm{i} k p \cos (p d) & \left(k^{2}-q^{2}\right) \cos (q d) \\
\left(k^{2}-q^{2}\right) \sin (p d) & 2 \mathrm{i} k q \sin (q d)
\end{array}\right]\left[\begin{array}{l}
A_{1} \\
B_{2}
\end{array}\right]=\left[\begin{array}{l}
0 \\
0
\end{array}\right]
$$


A uniform system of linear equations can be solved when its determinant is equal to zero:

$$
D_{A}=\left(k^{2}-q^{2}\right)^{2} \sin (p d) \cos (q d)+4 k^{2} p q \cos (p d) \sin (q d)=0
$$

After transformations:

$$
\frac{\tan (q d)}{\tan (p d)}=-\frac{\left(k^{2}-q^{2}\right)^{2}}{4 k^{2} p q} \quad \text { antisymmetric modes }
$$

One should note that $p$ and $q$ depend on the wavenumber $k$ as well as on the frequency $f=\omega / 2 \pi$. Equation (1.86) is known as the Rayleigh-Lamb frequency equation or the dispersion equation. Using this equation, one can compute the antisymmetric mode (A) velocity, with which waves propagate in a plate of thickness $d$ for a chosen frequency $f$. A numerical solution of Equation (1.86) is a set of antisymmetric eigenvalues $k_{0}^{A}, k_{1}^{A}, k_{2}^{A}, \ldots$ Substituting the eigenvalues into the uniform system of Equations (1.86) allows the coefficients $\left(A_{1}, B_{2}\right)$ to be determined in the form:

$$
A_{1}=2 \mathrm{i} k q \sin (q d), \quad B_{2}=-\left(k^{2}-q^{2}\right) \sin (p d)
$$

Substitution of the coefficients $\left(A_{1}, B_{2}\right)$ into Equations (1.71) yields antisymmetric modes of the Lamb waves:

$$
\begin{aligned}
& u_{x}^{A}=-2 k^{2} q \sin (q d) \sin (p y)+q\left(k^{2}-q^{2}\right) \sin (p d) \sin (q y) \\
& u_{y}^{A}=-\mathrm{i}\left[2 k p q \sin (q d) \cos (p y)+k\left(k^{2}-q^{2}\right) \sin (p d) \cos (q y)\right]
\end{aligned}
$$

Stress distribution is obtained by means of substituting the coefficients $\left(A_{1}, B_{2}\right)$ into Equations (1.71) and (1.73):

$$
\begin{aligned}
\sigma_{x x}^{A}= & -(2 \mathrm{i} k q)\left(\lambda p^{2}+(\lambda+2 \mu) k^{2}\right) \sin (q d) \sin (p y) \\
& +\mu\left(q^{2}-k^{2}\right) \sin (p d) \sin (q y) \\
\sigma_{y y}^{A}= & 2 \mu(\mathrm{i} k q)\left(k^{2}-q^{2}\right)[\sin (q d) \sin (p y)-\sin (p d) \sin (q y)] \\
\sigma_{y x}^{A}= & -\mu\left[4 k^{2} p q \sin (p d) \cos (p y)+\left(k^{2}-q^{2}\right)^{2} \sin (q d) \cos (p y)\right]
\end{aligned}
$$




\subsubsection{Numerical Solution of Rayleigh-Lamb Frequency Equations}

Solving the Rayleigh-Lamb frequency Equations (1.78) and (1.86) is not easy, because the parameters $p$ and $q$ are also dependent on the wavenumber. These equations can be analysed as relationships $\omega(k)$ or $c(\omega)$ describing dispersion curves, where $\omega$ is the angular frequency and $c$ is the phase velocity. The phase velocity is defined by the following formula:

$$
c=\frac{\omega}{k}
$$

For the given frequency there is an infinite number of solutions in the form of wavenumbers fulfilling Equations (1.78) and (1.86). These solutions can be real, imaginary or complex. However, in the case of the nonloaded plate problem it is sufficient to consider real wavenumber $k$ values only. This can be achieved by means of the following system of equations:

$$
\begin{array}{cc}
\frac{\tan (q d)}{q}+\frac{4 k^{2} p \tan (p d)}{\left(q^{2}-k^{2}\right)^{2}}=0 & \text { symmetric modes } \\
q \tan (q d)+\frac{\left(q^{2}-k^{2}\right)^{2} \tan (p d)}{4 k^{2} p}=0 & \text { antisymmetric modes }
\end{array}
$$

Further transformations aimed at introducing phase velocity and the product of frequency and thickness as analysis parameters lead to the following relationships:

$$
\begin{aligned}
L H^{S} & =\frac{\tan (\hat{q} \omega d)}{\hat{q}}+\frac{4 \hat{p} \tan (\hat{p} \omega d)}{c^{2}\left(\hat{q}^{2}-1 / c^{2}\right)^{2}} \\
L H^{A} S^{A} & =\hat{q} \tan (\hat{q} \omega d)+\frac{\left(\hat{q}^{2}-1 / c^{2}\right)^{2} \tan (\hat{p} \omega d)}{4 \hat{p} c^{2}}
\end{aligned}
$$

where:

$$
\omega d=2 \pi f d, \quad \hat{p}=\sqrt{\frac{1}{c_{L}^{2}}-\frac{1}{c^{2}}}, \quad \hat{q}=\sqrt{\frac{1}{c_{S}^{2}}-\frac{1}{c^{2}}}
$$

An algorithm that can be used for solving Equation (1.92) is presented below [4]: 
1. Choose an initial value of the product of frequency and thickness $(\omega d)_{0}$.

2. Estimate the value of the phase velocity $c_{0}$.

3. Investigate the signs of the left-hand sides $L H S^{S}$ and $L H S^{A}$ (assuming they are nonzero).

4. Repeat steps 3 and 4 until signs in one of the equations $L H S^{S}$ or $L H S^{A}$ change.

5. Use the bisection method to locate the value of phase velocity precisely within the $c_{n}<c<c_{n+1}$ range, where $n+1$ is the step at which sign changes occur.

6. Continue iterating until the left-hand side of the desired equation is close to zero.

7. After locating the root continue searching for the value of the frequency-thickness product $\omega d$, in order to locate other conceivable roots by repeating steps 2 to 6 .

8. Choose a different value of the $\omega d$ product and repeat steps 2 to 7 .

The procedure presented above is run for such number of $\omega d$ values as to achieve the desired accuracy.

One should note that despite the investigated functions being continuous, sign changes accompanying a zero crossing may pass unnoticed if increments of $\omega d$ are too large. This is due to the fact that the left-hand sides of Equations (1.91) have plots with peaks passing through zero in narrow ranges of the frequency-thickness product. That is why one needs to enhance the algorithm with an additional rule that would account for missed roots. This can be achieved by extrapolating the phase velocity curve. In case a root is lost, extrapolation allows the dispersion curve to be complemented with the missing root in the analysed frequency range. Sample results of root extraction from Equations (1.92) in the form of dispersion curves for an aluminium plate are presented in Figure 1.12. One should note that the procedure described above can be programmed in such a fashion that roots are classified into families of symmetric modes $S_{0}, S_{1}, S_{2}, \ldots$ and antisymmetric modes $A_{0}, A_{1}, A_{2}, \ldots$

From the shear horizontal mode (SHM) point of view, another important property of Lamb waves are the group velocity dispersion curves. Group velocity is defined as:

$$
c_{g}=\frac{\mathrm{d} \omega}{\mathrm{d} k}
$$

However, in order to reduce computation time and complexity of the code that computes dispersion curves, group velocity can be derived from phase 


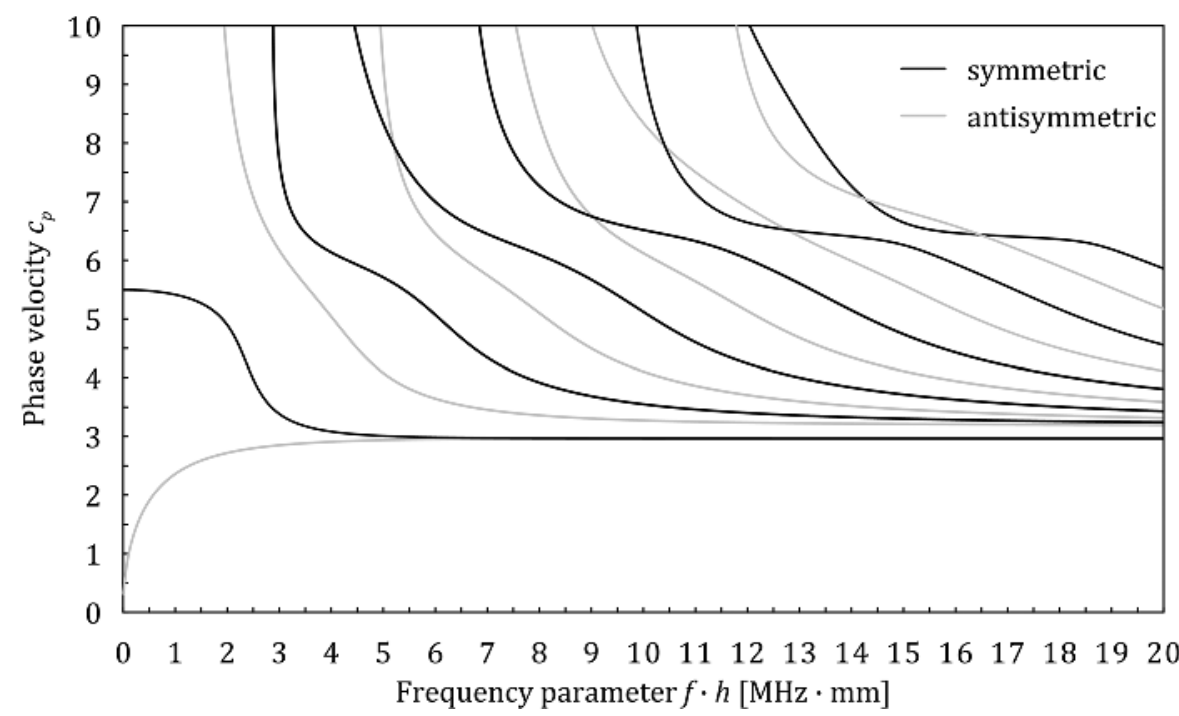

Figure 1.12 Phase velocity dispersion curves for symmetric and antisymmetric modes of Lamb waves ( $\left.c_{L}=6.3 \mathrm{~km} / \mathrm{s}, c_{S}=3.2 \mathrm{~km} / \mathrm{s}\right)$

velocity. After substituting $k=\omega / c$ into Equation (1.94) the group velocity is defined as [4]:

$$
c_{g}=\frac{\mathrm{d} \omega}{\mathrm{d}\left(\frac{\omega}{c}\right)}=\frac{\mathrm{d} \omega}{\frac{\mathrm{d} \omega}{c}-\omega \frac{\mathrm{d} c}{c^{2}}}=\frac{c^{2}}{c-\omega \frac{\mathrm{d} c}{\mathrm{~d} \omega}}
$$

After taking $\omega=2 \pi f$ into account, the third equality can be written as:

$$
c_{g}=\frac{c^{2}}{c-(f d) \frac{\mathrm{d} c}{\mathrm{~d}(f d)}}
$$

where $f d$ denotes the product of frequency and thickness. One should note that when the derivative of phase velocity with regard to $f d$ is equal to zero, then $c_{g}=c$. One should also note that when the derivative of phase velocity with regard to $f d$ approaches infinity (i.e. at the cut-off frequency), the group velocity approaches zero. Numerical derivation can be performed 


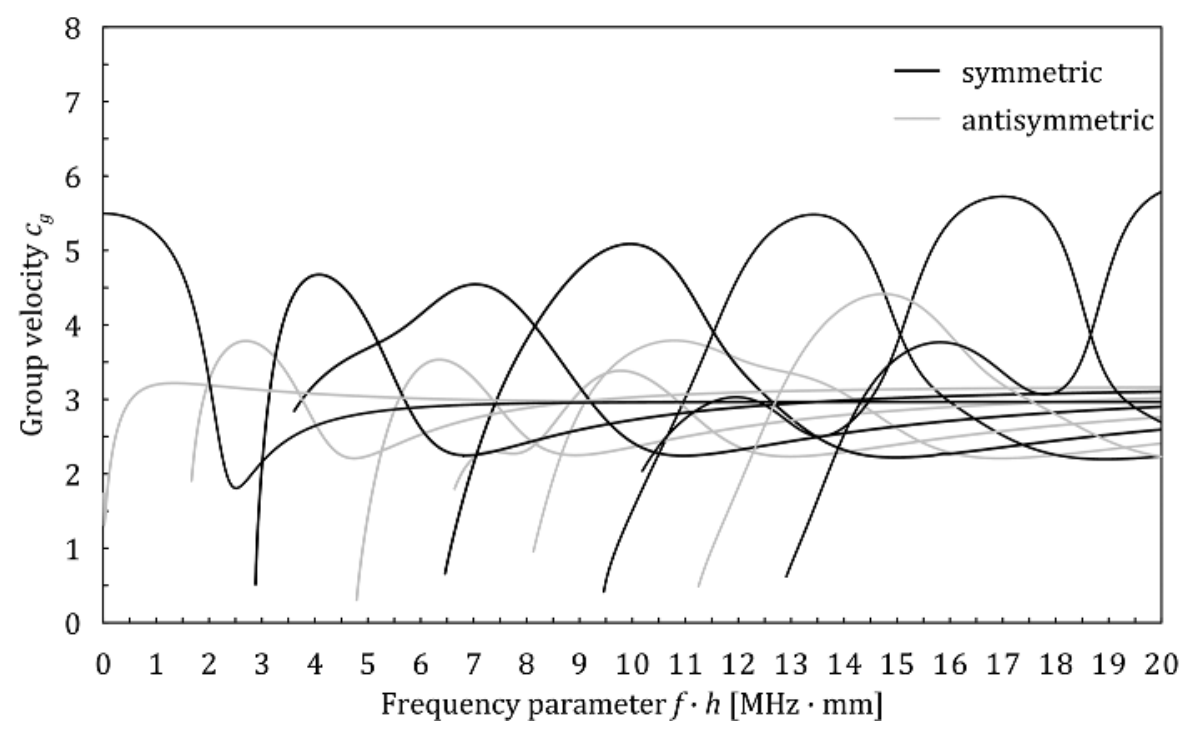

Figure 1.13 Group velocity dispersion curves for symmetric and antisymmetric modes of Lamb waves $\left(c_{L}=6.3 \mathrm{~km} / \mathrm{s}, c_{S}=3.2 \mathrm{~km} / \mathrm{s}\right)$

by applying the finite differences formula:

$$
\frac{\mathrm{d} c}{\mathrm{~d}(f d)} \cong \frac{\Delta c}{\Delta(f d)}
$$

Graphs of group velocity dispersion curves for an aluminium plate for symmetric and antisymmetric modes of Lamb waves are presented in Figure 1.13.

\subsubsection{Distribution of Displacements and Stresses for Various Frequencies of Lamb Waves}

After finding the roots of Equations (1.91) and classifying the dispersion curves one can compute the distributions of displacements and stress across the plate thickness according to Equations (1.80), (1.81), (1.88) and (1.89). Sample graphs of displacement and stress distributions across the plate thickness depending on frequency and type of Lamb wave mode are presented in Figures 1.14 and 1.15. It is evident that as the frequency rises, the distribution of displacements and stresses across the plate thickness becomes increasingly 


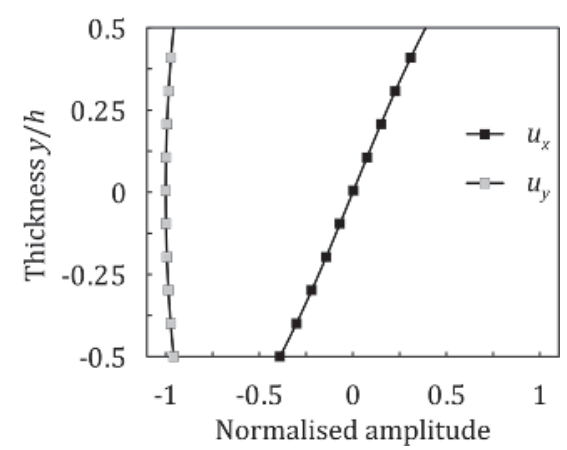

(a)

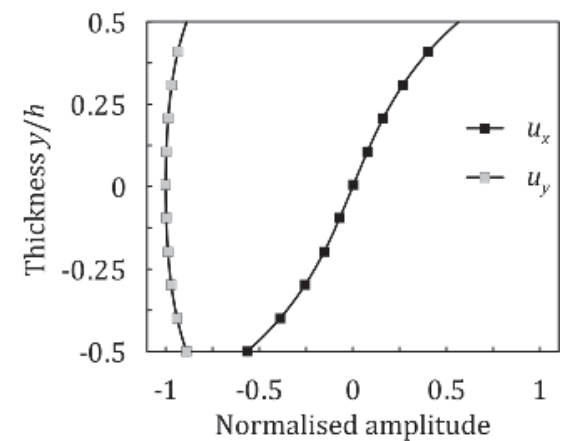

(c)

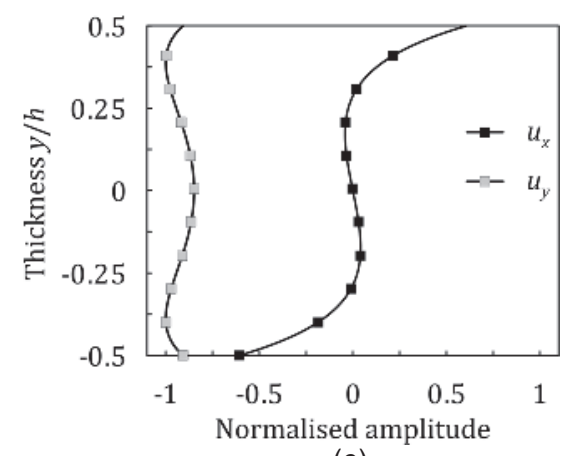

(e)

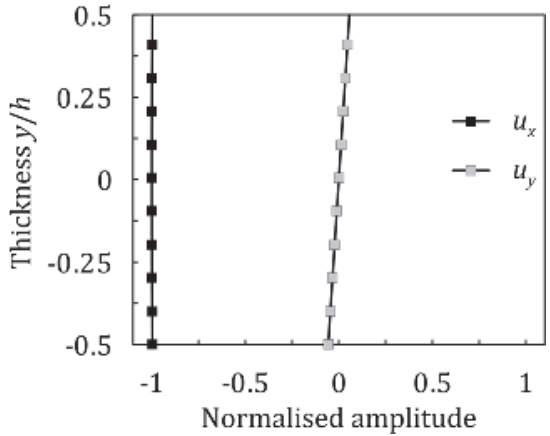

(b)

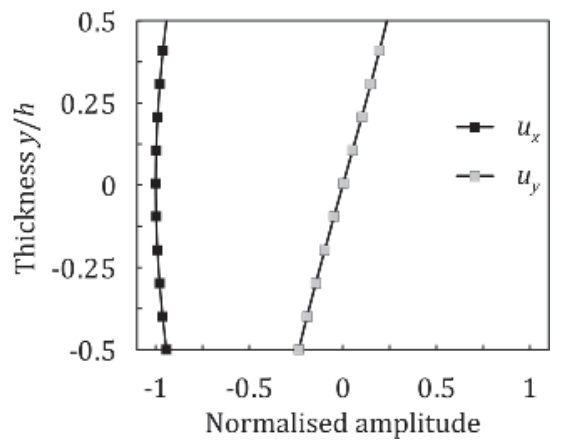

(d)

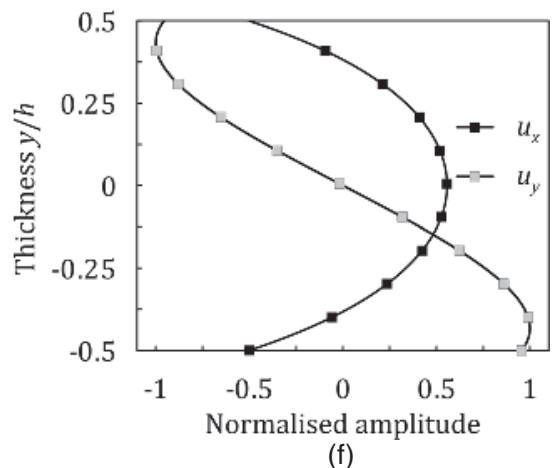

(f)

Figure 1.14 Distribution of longitudinal displacements $u_{x}$ and transverse displacements $u_{y}$ across the plate thickness for antisymmetric and symmetric modes of Lamb waves for individual frequencies. 


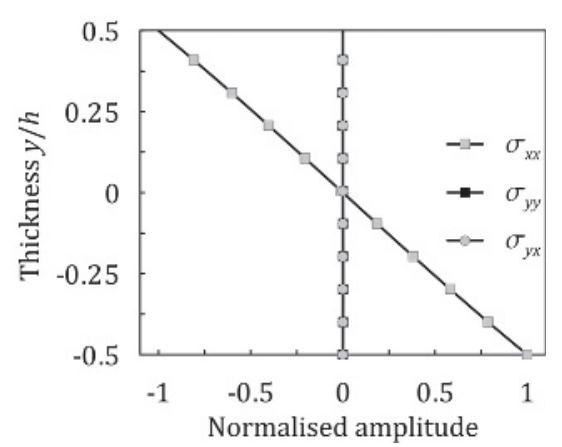

(a)

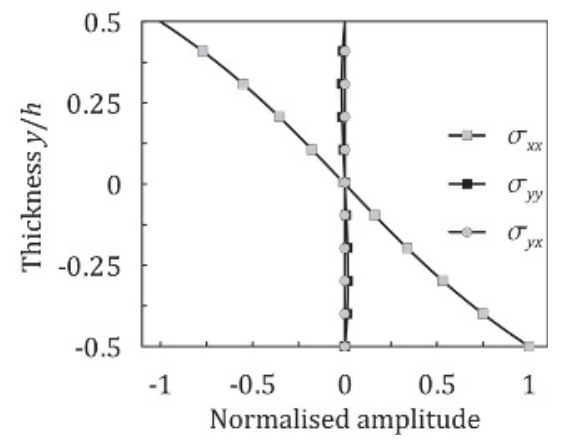

(c)

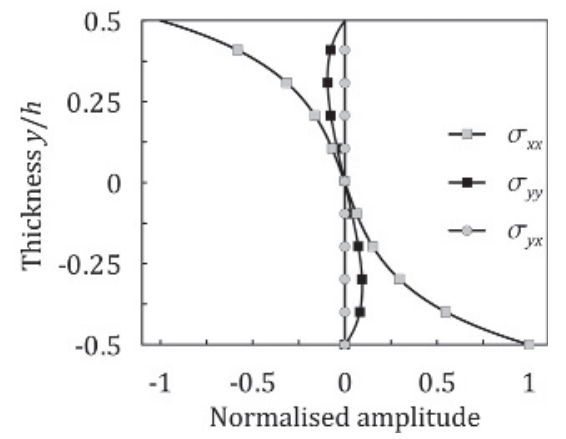

(e)

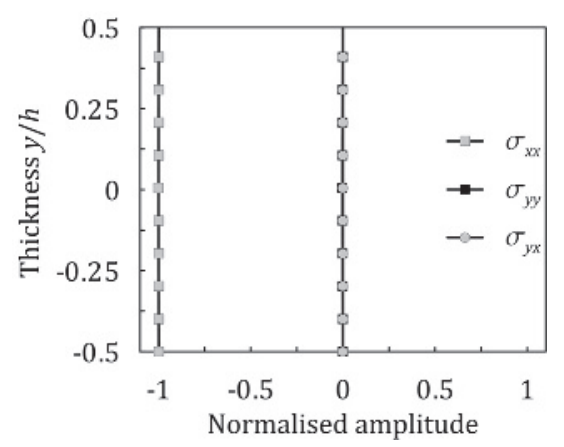

(b)

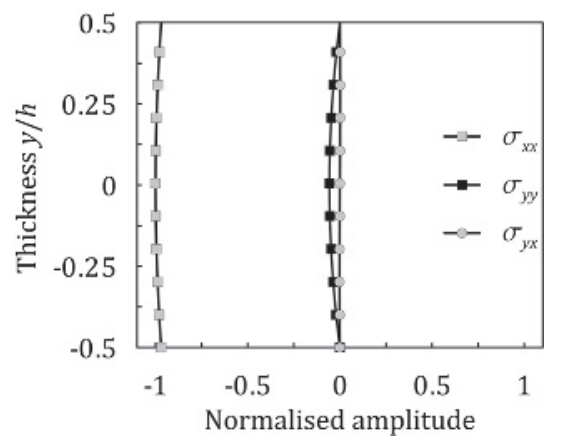

(d)

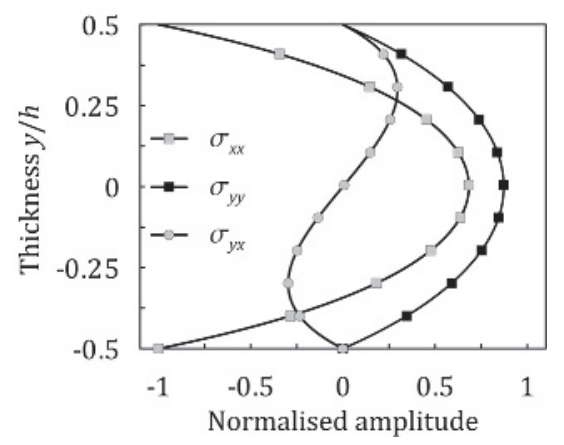

(f)

Figure 1.15 Distribution of stresses across plate thickness for antisymmetric and symmetric modes of Lamb waves for individual frequencies 
complex. In other words, for higher frequencies one must use polynomials of higher orders for fitting the curves.

\subsubsection{Shear Horizontal Waves}

Apart from Lamb wave modes that exist in flat plates, there also exist a set of time-harmonic wave motions known as shear horizontal (SH) modes. In case of shear horizontal waves the particle motion (displacements and velocities) are in a plane, that is parallel to the surface of the plate $(x, z)$ (see Figure 1.1). Physically, any mode in the SH family can be considered as the superposition of bulk waves reflecting from the upper and lower surfaces of the plate, polarized horizontally (in the $z$ axis direction). Particle motion has only a $u_{z}$ component and the wave Equation (1.47) simplifies to:

$$
\nabla^{2} u_{z}=\frac{1}{c_{S}^{2}} \ddot{u}_{z}
$$

It is assumed that the particle motion has the form:

$$
u_{z}(x, y, t)=h(y) \mathrm{e}^{\mathrm{i}(k x-\omega t)}
$$

The first part of Equation (1.99) represents a standing wave $h(y)$ across the plate thickness. The second part, $\mathrm{e}^{\mathrm{i}(k x-\omega t)}$, represents a wave propagating in the $x$ direction. Substitution of Equation (1.99) into Equation (1.98) and division of both sides by $\mathrm{e}^{\mathrm{i}(k x-\omega t)}$ yields:

$$
h^{\prime \prime}(y)+\eta^{2} h(y)=0
$$

where:

$$
\eta^{2}=\frac{\omega^{2}}{c_{S}^{2}}-k^{2}
$$

The solution of Equation (1.100) has the general form:

$$
h(y)=C_{1} \sin (\eta y)+C_{2} \cos (\eta y)
$$

Without going into detail, the tractions-free boundary conditions at the upper and lower plate surfaces:

$$
\sigma_{y z}(x, \pm d, t)=0
$$


leads to the system of linear homogeneous equations with the determinant:

$$
\sin (\eta d) \cos (\eta d)=0
$$

Equation (1.104) is the characteristic equation of $\mathrm{SH}$ wave modes and is zero when either:

$$
\sin (\eta d)=0
$$

which corresponds to symmetric modes of $\mathrm{SH}$ waves, or:

$$
\cos (\eta d)=0
$$

which corresponds to antisymmetric modes of $\mathrm{SH}$ waves.

Explicit solutions of Equations (1.105) and (1.106) are:

$$
\begin{aligned}
\eta^{S} d=n \pi, & n=0,1,2, \ldots \\
\eta^{A} d=(2 n+1) \frac{\pi}{2}, & n=0,1,2, \ldots
\end{aligned}
$$

for symmetric and antisymmetric modes, respectively. Finally, phase velocity dispersion curves of $\mathrm{SH}$ wave modes can be obtained from Equations (1.101) and (1.107) by recalling that $k=\omega / c$ :

$$
c(\omega)=\frac{c_{S}}{\sqrt{1-(\eta d)^{2}\left(\frac{c_{S}}{\omega d}\right)^{2}}}
$$

Phase velocity dispersion curves calculated according to Equation (1.108) are presented in Figure 1.17. It should be noted that the first symmetric SH wave mode is not dispersive because its eigenvalues is zero $\left(\eta_{0}^{S} d=0\right)$, and hence Equation (1.108) leads to $c^{S_{0}}(\omega)=c_{s}$. Figure 1.16 also indicates the asymptotic behaviour of the $\mathrm{SH}$ wave velocity. If $\omega \rightarrow \infty$, then $c \rightarrow c_{S}$.

It can be shown that the group velocity of $\mathrm{SH}$ waves is inversely proportional to the phase velocity and can be expressed as [5]:

$$
c_{g}(\omega)=c_{S} \sqrt{1-(\eta d)^{2}\left(\frac{c_{S}}{\omega d}\right)^{2}}
$$

Corresponding to Equation (1.109), the group velocity dispersion curves are presented in Figure 1.17. 


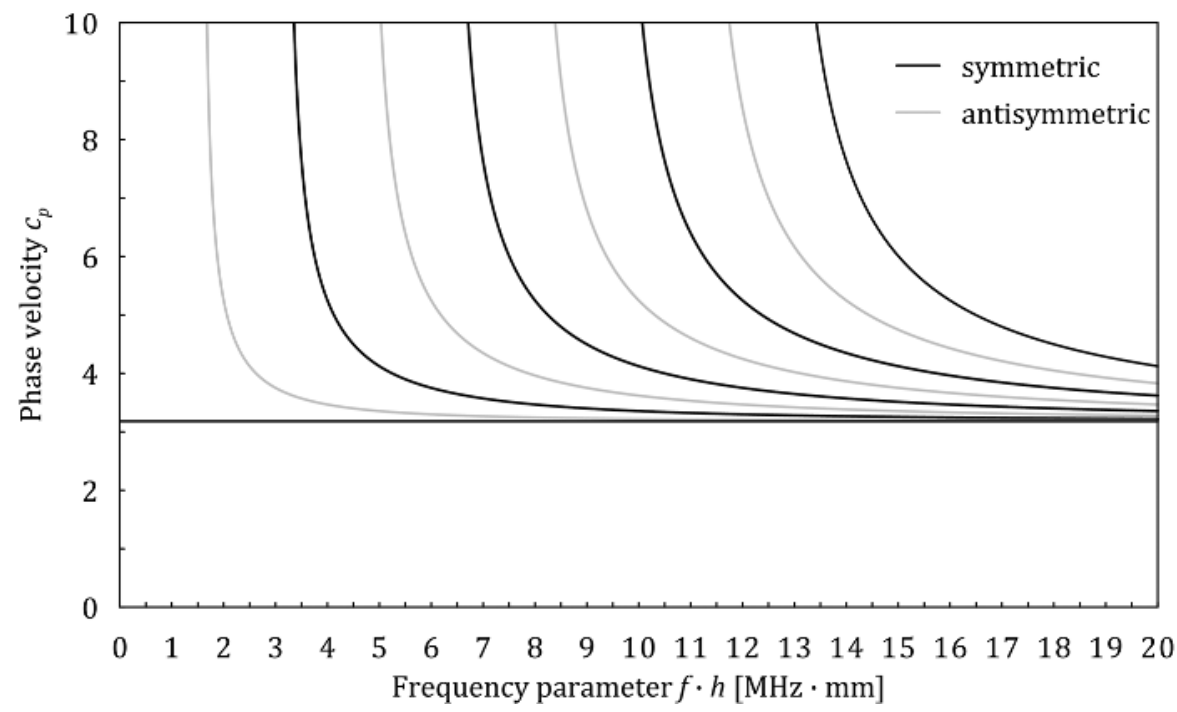

Figure 1.16 Phase velocity dispersion curves for symmetric and antisymmetric modes of $\mathrm{SH}$ waves ( $c_{S}=3.2 \mathrm{~km} / \mathrm{s}$ )

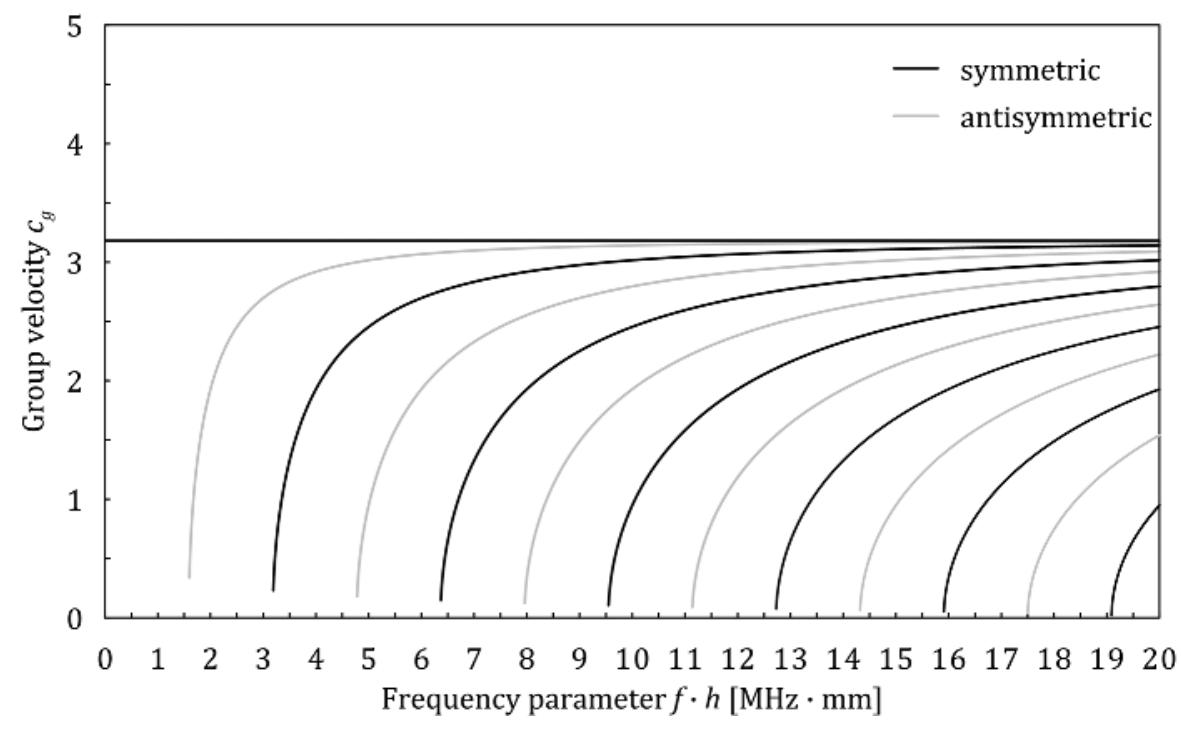

Figure 1.17 Group velocity dispersion curves for symmetric and antisymmetric modes of SH waves ( $c_{S}=3.2 \mathrm{~km} / \mathrm{s}$ ) 


\subsection{Wave Propagation in One-Dimensional Bodies of Circular Cross-Section}

\subsubsection{Equations of Motion}

Propagation of elastic waves in one-dimensional bodies is governed by equations of the linear theory of elasticity, which for isotropic media can be brought to tensor (1.17) or vector form:

$$
(\lambda+\mu) \nabla(\nabla \cdot \boldsymbol{u})+\mu \nabla^{2} \boldsymbol{u}=\rho \ddot{\boldsymbol{u}}
$$

The case of one-dimensional bodies of full circular cross-section is analysed here. It is most convenient to analyse this subject using the cylindrical system of coordinates $(x, r, \theta)$ instead of the Cartesian system $(x, y, z)$ (see Figure 1.18).

Using Helmholtz decomposition, one can express the displacement field vector $\boldsymbol{u}$ as the sum of the irrotational vector field $\boldsymbol{u}_{\phi}$ and the solenoidal vector field $\boldsymbol{u}_{r}$. This can be achieved by assuming that the displacement field vector is generated by a pair of potentials, that is scalar potential $\phi$ and vector

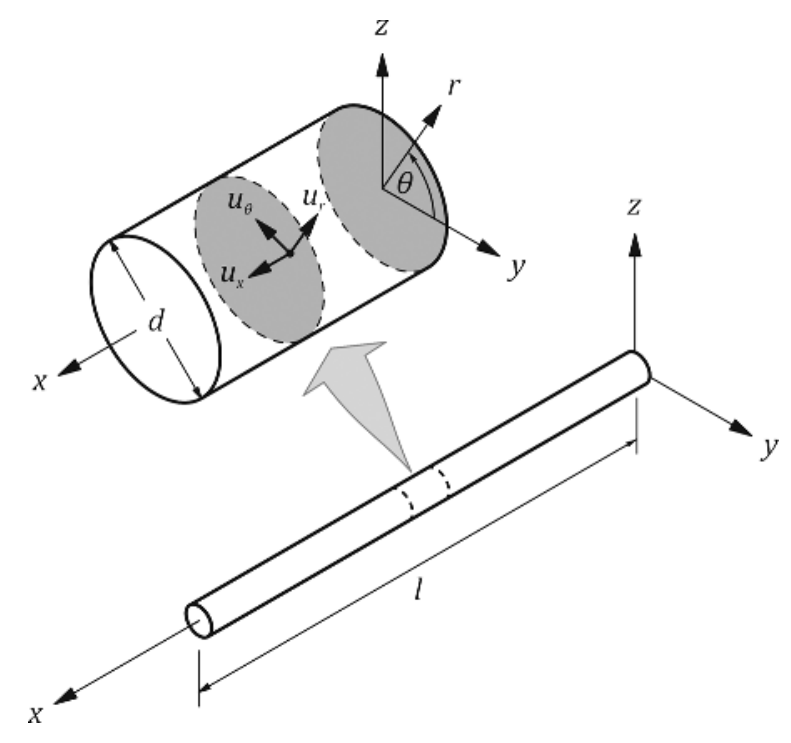

Figure 1.18 Shape of the structural rod element 
potential $\boldsymbol{H}=\left(H_{x}, H_{r}, H_{\theta}\right)$ :

$$
\boldsymbol{u}=\boldsymbol{u}_{\phi}+\boldsymbol{u}_{H}=\nabla \phi+\nabla \times \boldsymbol{H}, \quad \nabla \cdot \boldsymbol{H}=0
$$

using the following notation:

$$
\begin{aligned}
\nabla \phi= & i \frac{\partial \phi}{\partial x}+r \frac{\partial \phi}{\partial r}+\boldsymbol{\theta} \frac{1}{r} \frac{\partial \phi}{\partial \theta} \\
\nabla \cdot \boldsymbol{H}= & \frac{\partial H_{x}}{\partial x}+\frac{1}{r} \frac{\partial\left(r H_{r}\right)}{\partial r}+\frac{1}{r} \frac{\partial H_{\theta}}{\partial \theta} \\
\nabla \times \boldsymbol{H}= & \boldsymbol{i} \frac{1}{r}\left[\frac{\partial\left(r H_{\theta}\right)}{\partial r}-\frac{\partial H_{r}}{\partial \theta}\right]+r\left[\frac{1}{r} \frac{\partial H_{x}}{\partial \theta}-\frac{\partial H_{\theta}}{\partial x}\right] \\
& +\boldsymbol{\theta}\left[\frac{\partial H_{r}}{\partial x}-\frac{\partial H_{x}}{\partial r}\right] \\
\nabla^{2} u= & \frac{\partial^{2} u}{\partial x^{2}}+\frac{\partial^{2} u}{\partial r^{2}}+\frac{1}{r} \frac{\partial u}{\partial r}+\frac{1}{r^{2}} \frac{\partial^{2} u}{\partial \theta^{2}}
\end{aligned}
$$

where $\boldsymbol{i}, \boldsymbol{r}$ and $\boldsymbol{\theta}$ are unit vectors orientated along axes $x, r$ and $\theta$. Thus, displacement components can be expressed in the following form:

$$
\begin{aligned}
& u_{x}=\frac{\partial \phi}{\partial x}+\frac{1}{r} \frac{\partial\left(r H_{\theta}\right)}{\partial r}-\frac{1}{r} \frac{\partial H_{r}}{\partial \theta} \\
& u_{r}=\frac{\partial \phi}{\partial r}+\frac{1}{r} \frac{\partial H_{x}}{\partial \theta}-\frac{\partial H_{\theta}}{\partial x} \\
& u_{\theta}=\frac{1}{r} \frac{\partial \phi}{\partial \theta}+\frac{\partial H_{r}}{\partial x}-\frac{\partial H_{x}}{\partial r}
\end{aligned}
$$

Application of the Helmholtz theorem leads to motion equations identical with Equations (1.42) and (1.43), but formulated in the cylindrical system of coordinates:

$$
c_{L} \nabla^{2} \phi=\ddot{\phi}, \quad c_{S} \nabla^{2} \boldsymbol{H}=\ddot{\boldsymbol{H}}
$$

\subsubsection{Longitudinal Waves}

Analysis of longitudinal elastic waves in structural rod elements can be greatly simplified by the assumption of rotational symmetry of the rod with regard to the $x$ axis. Because of this symmetry all displacement and stress components must be independent of the $\theta$ angle. In the case of longitudinal waves the $u_{\theta}$ displacement component as well as $\gamma_{x \theta}$ and $\gamma_{r \theta}$ deformation 
components must be equal to zero, that is $u_{\theta}=\gamma_{x \theta}=\gamma_{r \theta}=0$. Moreover, one can demonstrate that a direct consequence of symmetry is that the potential vector $\boldsymbol{H}$ must have only one nonzero component $H_{\theta}$ and the other components $H_{x}$ and $H_{r}$ vanish, that is $H_{x}=H_{r}=0$ [4, 7]. Consequently, the nonzero components of the displacement vector in the rod can be expressed as:

$$
u_{x}=\frac{\partial \phi}{\partial x}+\frac{1}{r} \frac{\partial\left(r H_{\theta}\right)}{\partial r}, \quad u_{r}=\frac{\partial \phi}{\partial r}-\frac{\partial H_{\theta}}{\partial x}
$$

After substituting the relationships (1.115) into Equation (1.110) and simplifications, a system of two independent motion equations expressed using scalar potentials $\phi$ and $H_{\theta}$ is obtained:

$$
c_{L} \nabla^{2} \phi=\ddot{\phi}, \quad c_{S}\left(\nabla^{2} H_{\theta}-\frac{H_{\theta}}{r^{2}}\right)=\ddot{H}_{\theta}
$$

The second equation of this system can be simplified further, thanks to the fact that:

$$
\frac{\partial}{\partial r} \nabla^{2} H_{\theta}=\nabla^{2} \frac{\partial H_{\theta}}{\partial r}-\frac{1}{r^{2}} \frac{\partial H_{\theta}}{\partial r}
$$

Substituting:

$$
H_{\theta}=-\frac{\partial \psi}{\partial r}
$$

leads to:

$$
c_{L} \nabla^{2} \phi=\ddot{\phi}, \quad c_{S} \nabla^{2} \psi=\ddot{\psi}
$$

At the same time, components $u_{x}$ and $u_{r}$ of the displacement vector $u$ can be ultimately expressed as:

$$
u_{x}=\frac{\partial \phi}{\partial x}-\frac{\partial^{2} \psi}{\partial r^{2}}-\frac{1}{r} \frac{\partial \psi}{\partial r}, \quad u_{r}=\frac{\partial \phi}{\partial r}-\frac{\partial^{2} \psi}{\partial x \partial r}
$$

The displacement field in the rod can be easily computed on the basis of Equations (1.118). Nonzero components of the displacement field are 
as follows:

$$
\varepsilon_{x x}=\frac{\partial u_{x}}{\partial x}, \quad \varepsilon_{r r}=\frac{\partial u_{r}}{\partial r}, \quad \varepsilon_{\theta \theta}=\frac{u_{r}}{r}, \quad \gamma_{x r}=\frac{\partial u_{r}}{\partial x}+\frac{\partial u_{x}}{\partial r}
$$

while the stress field can be computed from Hooke's law, recalling the wellknown identities:

$$
\begin{aligned}
\sigma_{x x} & =2 \mu \varepsilon_{x x}+\lambda\left(\varepsilon_{x x}+\varepsilon_{r r}+\varepsilon_{\theta \theta}\right) \\
\sigma_{r r} & =2 \mu \varepsilon_{r r}+\lambda\left(\varepsilon_{x x}+\varepsilon_{r r}+\varepsilon_{\theta \theta}\right) \\
\sigma_{\theta \theta} & =2 \mu \varepsilon_{\theta \theta}+\lambda\left(\varepsilon_{x x}+\varepsilon_{r r}+\varepsilon_{\theta \theta}\right) \\
\tau_{x r} & =\mu \gamma_{x r}
\end{aligned}
$$

Harmonic waves propagating in the rod along the $x$ axis can be assumed as the solution of Equations (1.105) in a general complex form:

$$
\phi=\hat{\phi}(r) \mathrm{e}^{\mathrm{i}(k x-\omega t)}, \quad \psi=\hat{\psi}(r) \mathrm{e}^{\mathrm{i}(k x-\omega t)}
$$

where $\hat{\phi}(r)$ and $\hat{\psi}(r)$ are unknown functions. Substitution of the relationships (1.121) into the motion Equations (1.117) leads to a system of Bessel differential equations for functions $\hat{\phi}(r)$ and $\hat{\psi}(r)$ :

$$
\frac{\mathrm{d}^{2} \hat{\phi}}{\mathrm{d} r^{2}}+\frac{1}{r} \frac{\mathrm{d} \hat{\phi}}{\mathrm{d} r}+\alpha^{2} \hat{\phi}=0, \quad \frac{\mathrm{d}^{2} \hat{\psi}}{\mathrm{d} r^{2}}+\frac{1}{r} \frac{\mathrm{d} \hat{\psi}}{\mathrm{d} r}+\beta^{2} \hat{\psi}=0
$$

where:

$$
\alpha^{2}=\frac{\omega^{2}}{c_{L}^{2}}-k^{2}, \quad \beta^{2}=\frac{\omega^{2}}{c_{S}^{2}}-k^{2}
$$

which have solutions in the form of Bessel functions of the first type: $J_{0}(\alpha r)$ and $J_{0}(\beta r)$, as well as of the second type: $Y_{0}(\alpha r)$ and $Y_{0}(\beta r)$. As Bessel functions of the second type exhibit a singularity in the origin $r=0$, this branch of solutions is discarded, leading to the following form of solutions of the problem being analysed:

$$
\hat{\phi}=A J_{0}(\alpha r), \quad \hat{\psi}=B J_{0}(\beta r)
$$

where $A$ and $B$ are some constants. 
Taking into account the general form of solutions given as Equation (1.121), one can finally write that:

$$
\phi=A J_{0}(\alpha r) \mathrm{e}^{\mathrm{i}(k x-\omega t)}, \quad \psi=B J_{0}(\beta r) \mathrm{e}^{\mathrm{i}(k x-\omega t)}
$$

Propagation of longitudinal elastic waves in a rod requires meeting the boundary conditions of stresses vanishing on the external rod surface, which accompany the motion equation system (1.117):

$$
\sigma_{r r}(x, r)=\tau_{x r}(x, r)=0, \quad \text { dla } \quad 0 \leq x \leq l, \quad r=a=\frac{d}{2}
$$

where $l$ is the length and $d$ is the rod diameter.

After substituting Equations (1.124) into Equations (1.119), using the identities of Equations (1.120) again and some simplifications, the boundary conditions of the vanishing stress components $\sigma_{r r}$ and $\tau_{x r}$ lead to a system of two uniform equations expressed through the solutions from formulas (1.124).

A given system of equations has a nontrivial solution only if its determinant vanishes. In the analysed case this condition leads directly to a certain nonlinear equation known in literature as the Pochhammer frequency equation for longitudinal modes propagating in rods; this equation relates the angular frequency $\omega$ with wavenumber $k$. The Pochhammer frequency equation has the following form:

$$
\begin{aligned}
& \frac{2 \alpha}{a}\left(\beta^{2}+k^{2}\right) J_{1}(\alpha a) J_{1}(\beta a)-\left(\beta^{2}-k^{2}\right) J_{0}(\alpha a) J_{1}(\beta a) \\
& \quad-4 k^{2} \alpha \beta J_{1}(\alpha a) J_{0}(\beta a)=0
\end{aligned}
$$

It is worth noting that this equation was derived for the first time in 1876 by a Prussian mathematician Leo Pochhammer [8], who studied vibrations of circular cylinders. This equation was also studied by many other researchers (e.g. Chree [9], Love [10], Davis [11], Pao and Mindlin [12] and Graff [13]), but due to its complexity its roots remained unknown for many years.

\subsubsection{Solution of Pochhammer Frequency Equation}

In the analysed case Pochhammer frequency equation was solved using original dedicated software developed by M. Krawczuk and A. Żak for the MATLAB ${ }^{\circledR}$ environment [14]. Phase velocity and group velocity values for waves propagating in the rod were calculated under the assumption that the rod was made of aluminium alloy of Young's modulus $E=72.7 \mathrm{GPa}$, Poisson's coefficient $v=0.33$, material density $\rho=2700 \mathrm{~kg} / \mathrm{m}^{3}$ and diameter 
$d=0.01 \mathrm{~m}$. Characteristic velocities were $c_{L}=6.3 \mathrm{~km} / \mathrm{s}$ and $c_{S}=3.2 \mathrm{~km} / \mathrm{s}$, respectively.

The calculation range was set out by a frequency range from $0.1 \mathrm{~Hz}$ to 20 $\mathrm{MHz}$ and a phase frequency range $c$ from $2 \mathrm{~km} / \mathrm{s}$ to $50 \mathrm{~km} / \mathrm{s}$. Roots of the Pochhammer frequency equation were sought in nodes of a regular grid of $400 \times 2000$ nodes with an assumed accuracy of $\delta \leq 0.001 \%$.

Solving the equation involves applying the conjugate bisection method [15]. In the first step, roots were located as a function of the phase velocity $c_{p}=\omega / k$ for the given frequency, which was treated like a parameter in Equation (1.126). In the second step, the phase velocity $c$ was considered as a parameter and the roots were located as a function of frequency $f$. In this way the second step of calculations improved the solution obtained in the first step for those of the analysed areas where phase velocity changes were very large. Group velocity values were also computed numerically by derivation of the wavenumber curves $k=k(\omega)$ with regard to the angular frequency $\omega$.

Results obtained for changes of the group velocity to phase velocity ratio $c_{g} / c_{p}$ as a function of the parameter $f d$, where $f$ is frequency and $d$ is rod diameter, are shown in Figure 1.19. As can be seen in Figure 1.20, the phase

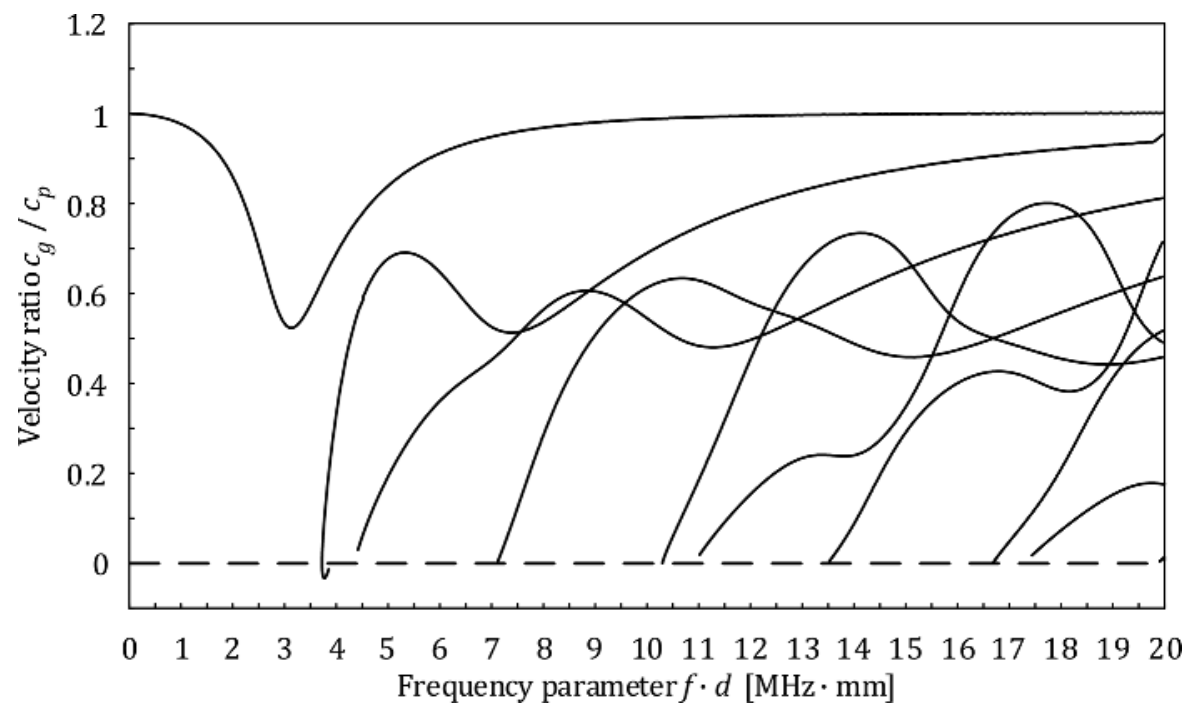

Figure 1.19 Dispersion curves of the group velocity to phase velocity ratio for the case of longitudinal modes in an aluminium rod $\left(c_{L}=6.3 \mathrm{~km} / \mathrm{s}, c_{S}=\right.$ $3.2 \mathrm{~km} / \mathrm{s}$ ) 


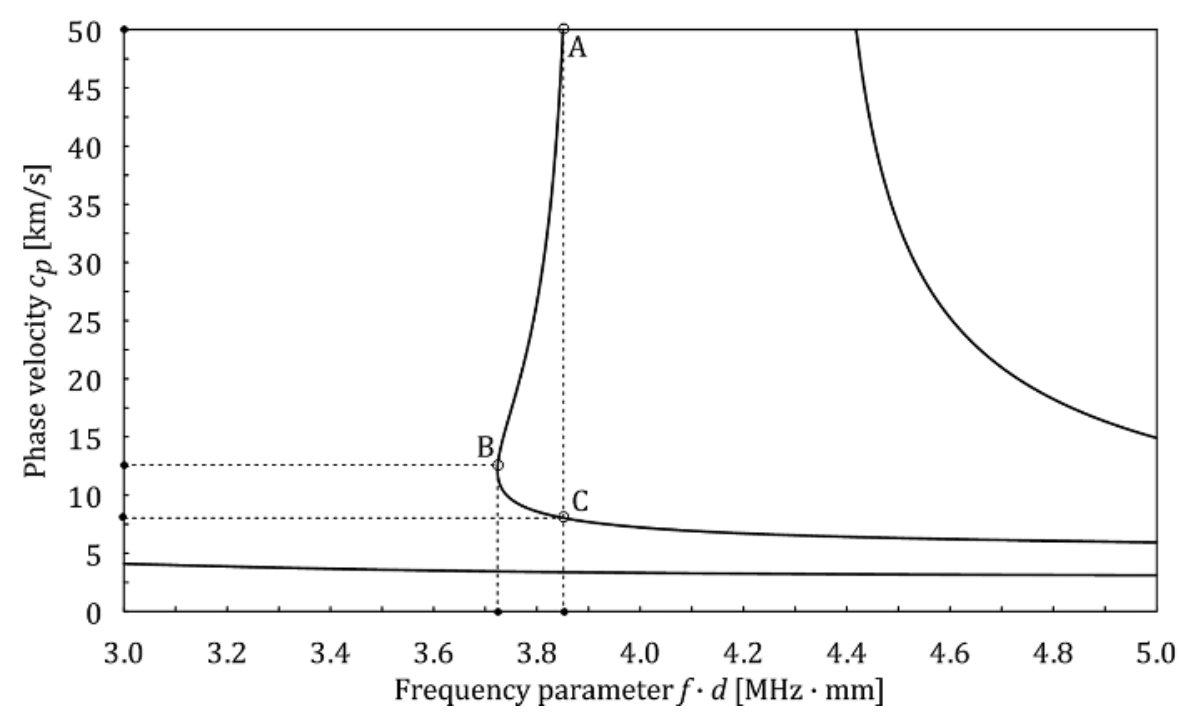

Figure 1.20 Increase of the second mode of the phase velocity dispersion curve for an aluminium rod

velocity dispersion curve for the second mode exhibits very unusual behaviour near the cut-off frequency for this mode between points $A, B$ and $C$. One can see that between points $A$ and $B$ the group velocity and the phase velocity have opposite signs. This suggests that the direction of energy transfer in the rod may be opposite to the wave propagation direction. In other words, wave motion carries energy in one direction, but wave propagation seems to occur in the other direction. This phenomenon, called wave backpropagation, was investigated and documented in the literature by many researchers (e.g. Meeker and Meitzler [16], Meitzler [17], Alippi et al. [18] and Marston [19]) and still is the subject of research, especially in the case of electromagnetic waves.

In the frequency parameter $f d$ range from a cut-off frequency of $3.72 \mathrm{MHz} \cdot \mathrm{mm}$ (point B) to $3.85 \mathrm{MHz} \cdot \mathrm{mm}$ (points A and C) the phase velocity curve $c_{p}$ takes double values, which indicates two different zones of group velocity $c_{g}$ values. The first branch of the phase velocity curve $c_{p}=c_{p}(f d)$ between points $A$ and $B$ is an area of high phase velocities, where phase velocity $c_{p}$ and group velocity $c_{g}$ have opposite signs. The second branch between points $B$ and $C$ is an area of low phase velocities, where phase velocity $c_{p}$ and group velocity $c_{g}$ have the same signs. 


\subsubsection{Torsional Waves}

Torsional waves are a consequence of vanishing displacements $u_{r}$ and $u_{z}$. Because of rotational symmetry, displacement $u_{\theta}$ must be independent of $\theta$. For torsional waves the motion equation is as follows [13]:

$$
\frac{\partial^{2} u_{\theta}}{\partial r^{2}}+\frac{1}{r} \frac{\partial u_{\theta}}{\partial r}-\frac{u_{\theta}}{r}+\frac{\partial u_{\theta}}{\partial x^{2}}=\frac{1}{c_{S}^{2}} \frac{\partial^{2} u_{\theta}}{\partial t^{2}}
$$

Harmonic waves of the following form are assumed:

$$
u_{\theta}=V(r) \mathrm{e}^{\mathrm{i}(k x-\omega t)}
$$

Substituting Equation (1.128) into Equation (1.127) and solving the differential equation for the unknown function $V(r)$ leads to:

$$
u_{\theta}=\frac{1}{\beta} B J_{1}(\beta r) \mathrm{e}^{\mathrm{i}(k x-\omega t)}
$$

where $B$ is any constant.

From the boundary conditions:

$$
\sigma_{r r}(x, r)=\tau_{x r}(x, r)=\tau_{r \theta}(x, r)=0, \quad \text { for } \quad 0 \leq x \leq l, \quad r=a=\frac{d}{2}
$$

the only nontrivial condition is the following one:

$$
\tau_{r \theta}(x, r)=0, \quad \text { for } \quad 0 \leq x \leq l, \quad r=a=\frac{d}{2}
$$

This condition leads to a dispersion equation for torsional modes propagating in rods that relates the angular frequency $\omega$ with wavenumber $k$ :

$$
(\beta a) J_{0}(\beta a)-2 J_{1}(\beta a)=0
$$

The first three roots of Equation (1.132) are:

$$
\beta_{1}=0, \quad \beta_{2} a=5.136, \quad \beta_{3} a=8.417
$$


One should note that $\beta=0$ is also a solution of the dispersion equation. The limit transition $\beta \rightarrow 0$ in Equation (1.129) leads to the following equation:

$$
u_{\theta}=\frac{1}{2} B r \mathrm{e}^{\mathrm{i}(k x-\omega t)}
$$

This displacement represents the lowest torsional mode. In the lowest mode displacement the amplitude $u_{\theta}$ is proportional to the radius. The motion corresponding to the solution is rotation of each rod cross-section as a whole around its centre. One should note that $\beta=0$, which implies that the phase velocity is equal to the shear wave velocity $c_{S}$ :

$$
\beta^{2}=\frac{\omega^{2}}{c_{S}^{2}}-k^{2}, \quad \beta=0, \quad \rightarrow \quad c_{S}=\frac{\omega}{k}=c
$$

Thus the lowest torsional mode is nondispersive. Higher modes are dispersive. For the given frequency, solutions of Equation (1.132) in the form of wavenumbers $k_{n}$ can take real or imaginary values. For real values the $k_{n}(\omega)$ branches are hyperbolically shaped, while for imaginary values they are circles.

\subsubsection{Flexural Waves}

Flexural waves are dependent on the circumferential angle $\theta$ and in the displacement vector all three components are nonzero and change according to simple trigonometry-based relationships:

$$
\begin{aligned}
& u_{x}=U_{x}(r) \cos \theta \mathrm{e}^{\mathrm{i}(k x-\omega t)} \\
& u_{r}=U_{r}(r) \sin \theta \mathrm{e}^{\mathrm{i}(k x-\omega t)} \\
& u_{\theta}=U_{\theta}(r) \cos \theta \mathrm{e}^{\mathrm{i}(k x-\omega t)}
\end{aligned}
$$

After substituting the displacement components (1.134) into the system of displacement Equations (1.114) a system of three ordinary differential equations containing the functions $U_{x}, U_{y}$ and $U_{\theta}$ is obtained. Without going into 
the details of solving these equations, their ultimate form is as follows:

$$
\begin{aligned}
& U_{x}(r)=\mathrm{i} k A J_{1}(\alpha r)-\frac{C}{r} \frac{\partial}{\partial r}\left[r J_{2}(\beta r)\right]-\frac{C}{r} J_{2}(\beta r) \\
& U_{r}(r)=A \frac{\partial}{\partial r} J_{1}(\alpha r)+\frac{B}{r} J_{1}(\beta r)+\mathrm{i} k C J_{2}(\beta r) \\
& U_{\theta}(r)=-\frac{A}{r} J_{1}(\alpha r)+\mathrm{i} k C J_{2}(\beta r)-B \frac{\partial}{\partial r} J_{1}(\beta r)
\end{aligned}
$$

Particular integrals of (1.135) are chosen in such fashion that they do not have singularities on the rod axis. From the conditions of zero stresses on the cylinder surface:

$$
\sigma_{r r}(x, r)=\tau_{x r}(x, r)=\tau_{r \theta}(x, r)=0, \quad \text { for } \quad 0 \leq x \leq l, \quad r=a=\frac{d}{2}
$$

after applying the relationships (1.135) a system of three equations [20] is obtained. This is a system of equations uniform with regard to constants $A, B$ and $C$. The condition of the vanishing system determinant leads to a frequency equation [13], from which one can determine subsequent roots $k$.

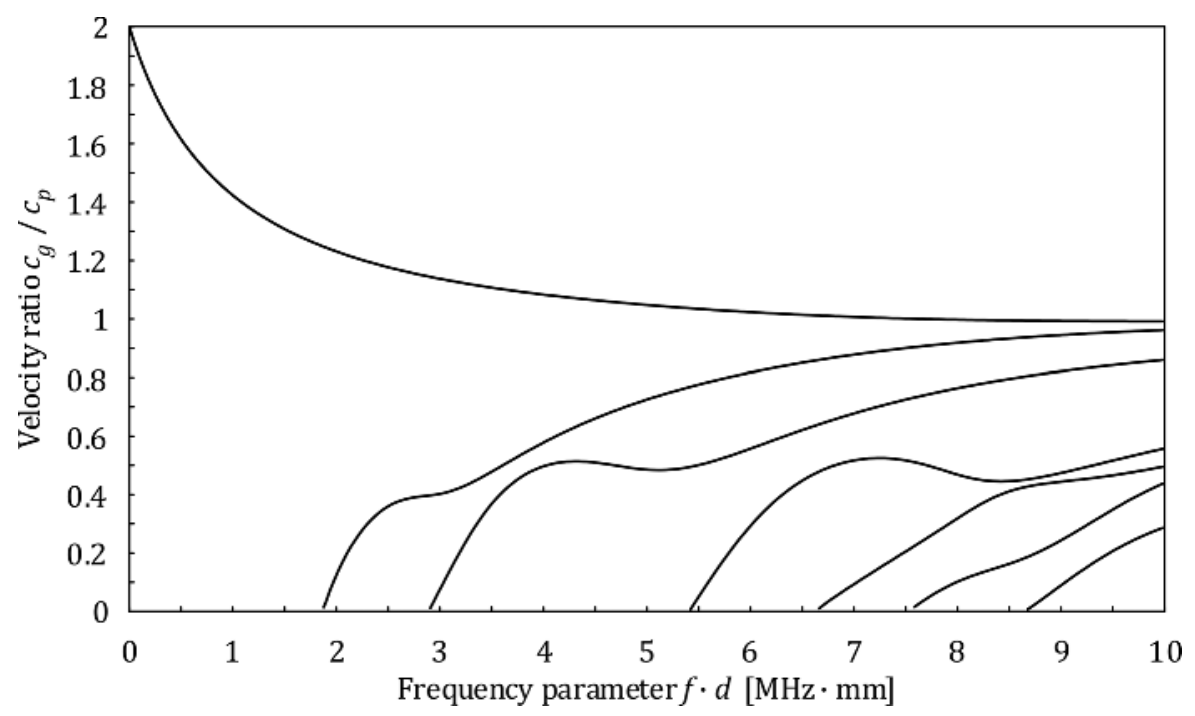

Figure 1.21 Dispersion curves of the group velocity to phase velocity ratio for the case of flexural modes in an aluminium rod $\left(c_{L}=6.3 \mathrm{~km} / \mathrm{s}, c_{S}=3.2 \mathrm{~km} / \mathrm{s}\right)$ 
The frequency equation for flexural waves in rods of circular cross-section was investigated by Pao and Mindlin [12].

Results obtained for changes of the group velocity to phase velocity ratio $c_{g} / c_{p}$ as a function of the parameter $f d$, where $f$ is frequency and $d$ is rod diameter, are shown in Figure 1.21.

\section{References}

1. Lamb, H. (1917) On waves in an elastic plate. Proceedings of the Royal Society of London, 93, 293-312.

2. Worlton, D.C. (1961) Experimental confirmation of Lamb waves at megacycle frequencies, Journal of Applied Physics, 32, 967-971.

3. Kolsky, H. (1963) Stress Waves in Solids, Dover Publications, Inc., New York.

4. Rose, J.L. (1999) Ultrasonic Waves in Solid Media, Cambridge University Press, Cambridge.

5. Giurgiutiu, V. (2007) Structural Health Monitoring with Piezoelectric Wafer Active Sensors, Academic Press.

6. Doyle, J.F. (1997) Wave Propagation in Structures, Springer-Verlag New York, Inc., New York.

7. Achenbach, J.D. (1973) Wave Propagation in Elastic Solids, North-Holland Publishing Company, Amsterdam.

8. Pochhammer, L. (1876) Biegung des Kreiscylinders - FortpflanzungsGeschwindigkeit Kleiner Schwingungen in einem Kreiscylinder. Journal für die reine und angewandte Mathematik, 81, 33-61.

9. Chree, C. (1889) The equations of an isotropic elastic solid in polar and cylindrical coordinates, their solutions and applications. Proceedings of the Cambridge Philosophical Society. Mathematical and Physical Sciences, 14, 250-369.

10. Love, A.E. (1927) A Treatise on the Mathematical Theory of Elasticity, 4th edn, Dover Publications, New York, Dover.

11. Davis, R.M. (1948) A critical study of the Hopkinson pressure bar. Philosophical Transactions of the Royal Society of London. Series A, Mathematical and Physical Sciences, 240, 375-457.

12. Pao, Y.H. and Mindlin, R.D. (1960) Dispersion of flexural waves in an elastic, circular cylinder. Journal of Applied Mechanics, 27, 513-520.

13. Graff, K.F. (1991) Wave Motion in Elastic Solids, Dover Publications, New York, Dover.

14. Żak, A. and Krawczuk M. (2010) Assessment of rod behaviour theories used in spectral finite element modelling. Journal of Sound and Vibration, 329(11), 2099-2113.

15. Ralston, A. (1965) A First Course in Numerical Analysis, McGraw-Hill Book Company, New York. 
16. Meeker, T.R. and Meitzler A.H. (1964) Guided wave propagation in elongated cylinders and plates, Chapter 2, in Physical Acoustics, vol. 1, Part A, Academic Press, New York.

17. Meitzler, A.H. (1965) Backward wave transmission of stress pulses in elastic cylinders and plates. The Journal of Acoustical Society of America, 38, 835-842.

18. Alippi, A., Bettucci, A. and Germano, M. (2000) Anomalous propagation characteristics of evanescent waves. Ultrasonics, 38, 817-820.

19. Marston, P.L. (2003) Negative group velocity Lamb waves on plates and applications to the scattering of sound by shells. The Journal of the Acoustical Society of America, 113, 2659-2662.

20. Bancroft, D. (1941) The velocity of longitudinal waves in cylindrical bars. Physical Review, 59, 588-593. 